\title{
HYPERKÄHLER MANIFOLDS WITH TORSION, SUPERSYMMETRY AND HODGE THEORY *
}

\author{
MISHA VERBITSKY ${ }^{\dagger}$
}

\begin{abstract}
Let $M$ be a hypercomplex Hermitian manifold, $(M, I)$ the same manifold considered as a complex Hermitian with a complex structure $I$ induced by the quaternions. The standard linearalgebraic construction produces a canonical nowhere degenerate $(2,0)$-form $\Omega$ on $(M, I)$. It is well known that $M$ is hyperkähler if and only if the form $\Omega$ is closed. The $M$ is called HKT (hyperkähler with torsion) if $\Omega$ is closed with respect to the Dolbeault differential $\partial: \Lambda^{2,0}(M, I) \longrightarrow \Lambda^{3,0}(M, I)$. Conjecturally, all compact hypercomplex manifolds admit an HKT-metrics. We exploit a remarkable analogy between the de Rham DG-algebra of a Kähler manifold and the Dolbeault DG-algebra of an HKT-manifold. The supersymmetry of a Kähler manifold $X$ is given by an action of an 8dimensional Lie superalgebra $\mathfrak{g}$ on $\Lambda^{*}(X)$, containing the Lefschetz $S L(2)$-triple, the Laplacian and the de Rham differential. We establish the action of $\mathfrak{g}$ on the Dolbeault DG-algebra $\Lambda^{*, 0}(M, I)$ of an HKT-manifold. This is used to construct a canonical Lefschetz-type $S L(2)$-action on the space of harmonic spinors of $M$.
\end{abstract}

1. Introduction. Hyperkähler manifolds with torsion (HKT-manifolds) were introduced by P.S.Howe and G.Papadopoulos ([HP]) were much studied in physics literature since then. For an excellent survey of these works written from a mathematician's point of view, the reader is referred to the paper of G. Grantcharov and Y. S. Poon [GP]. In physics, HKT-manifolds appear as moduli of brane solitons in supergravity and M-theory ([GP2], [P]). HKT-manifolds also arise as moduli space of some special black holes in $\mathrm{N}=2$ supergravity ([GP1], [GPS]).

The term "hyperkähler manifold with torsion" is actually quite misleading, because an HKT-manifold is not hyperkähler. This is why we prefer to use the abbreviation "HKT-manifold".

1.1. Hypercomplex manifolds. Let $M$ be a smooth manifold equipped with an action of quaternion algebra on its tangent bundle. The manifold $M$ is called hypercomplex ([Bo]) if for any quaternion $L \in \mathbb{H}, L^{2}=-1$, the corresponding almost complex structure is integrable. If, in addition, $M$ admits a Riemannian structure, and for any quaternion $L \in \mathbb{H}, L^{2}=-1, L$ establishes a Kähler structure on $M$, the manifold $M$ is called hyperkähler.

The geometry of hypercomplex manifold is quite rich, but not completely understood yet. There are compact homogeneous examples ([J]), compact inhomogeneous examples ([PP]). The task of producing new examples of compact hypercomplex manifolds is clearly not as difficult as that for compact hyperkähler manifolds.

The main tool of hypercomplex geometry is the so-called Obata connection. Given a hypercomplex manifold $M$, there exists a unique torsion-free connection on the tangent bundle $T M$ which preserves the quaternion action; this connection was introduced by M. Obata in 1950-ies ([O]). Clearly, the holonomy group of the Obata connection lies in $G L(n, \mathbb{H})$, where $n=\operatorname{dim}_{\mathbb{H}} M$. The manifold $M$ admits a hyperkähler metrics if and only if its holonomy preserves a positive definite metrics.

There are many results on deformations and Dolbeault cohomology of a homogeneous hypercomplex manifold ([PP], [GPP], etc.) However, the algebraic geometry of a general hypercomplex manifold is terra incognita, so far.

${ }^{*}$ Received July 12, 2002; accepted for publication September 25, 2002.

$\dagger$ 11-1-83 Orekhovy blvrd, Moscow 115551, Russia and 15 University Gardens, Glasgow G12 8QW, United Kingdom (verbit@dnttm.rssi.ru; verbit@mccme.ru). The author is partially supported by CRDF grant RM1-2087. 
Since these manifolds are (usually) not Kähler, no relation between the de Rham and Dolbeault cohomology is established. This is why the most natural geometrical questions are so difficult to solve.

1.2. HKT-metrics on hypercomplex manifolds. For a historically correct definition of an HKT-manifold ([HP]), see Subsection 3.2. However, throughout this paper, we use not this definition, but its reformulation, introduced by Grantcharov and Poon ([GP]).

Let $M$ be a hypercomplex manifold, and $h$ a Riemannian metrics on $M$. The metrics $h$ is called quaternionic Hermitian if $h$ is invariant with respect to the group $S U(2) \subset \mathbb{H}^{*}$ of unitary quaternions. In this case, $M$ is called a hypercomplex Hermitian manifold (Definition 2.5).

Given a hypercomplex Hermitian manifold, and an induced complex structure $L \in \mathbb{H}, L^{2}=-1$, one may consider the corresponding real-valued $(1,1)$-form $\omega_{L} \in$ $\Lambda^{1,1}(M, L), \omega_{L}(x, y):=h(x, L y)$. This is a well known anti-symmetric form associated with the Hermitian structure on a complex manifold $(M, L)$.

Let $I, J, K \in \mathbb{H}$ be the standard basis in quaternions. Consider the differential form $\Omega:=\frac{1}{2}\left(\omega_{J}+\sqrt{-1} \omega_{K}\right)$. An elementary linear-algebraic calculation ensures that $\Omega$ is of type $(2,0)$ with respect to the complex structure $I$ :

$$
\Omega \in \Lambda^{2,0}(M, I) .
$$

The following lemma is due to Grantcharov and Poon (it is a reformulation of Proposition 2 of [GP]).

Lemma 1.1. Let $M$ be a hypercomplex Hermitian manifold. Consider the subspace

$$
V \subset \Lambda^{3}(M)
$$

generated by 3 -forms of type

$$
d \omega_{L}, \quad L \in \mathbb{H}, \quad L^{2}=-1
$$

The space $\Lambda^{3}(M)$ is equipped with a natural action of the group of unitary quaternions $S U(2) \subset \mathbb{H}^{*}$. Let $I, J, K \in \mathbb{H}$ be the standard triple of quaternions. Then the following conditions are equivalent.

(i) The space $V$ belongs to a direct sum of several irreducible $S U(2)$-subresentations $V_{i} \subset \Lambda^{3}(M)$ of dimension $2 .^{1}$

(ii) We have $\partial \Omega=0$, where $\Omega \in \Lambda^{2,0}(M, I), \Omega:=\frac{1}{2}\left(\omega_{J}+\sqrt{-1} \omega_{K}\right)$ is the $(2,0)$ form constructed above, and $\partial: \Lambda^{2,0}(M, I) \longrightarrow \Lambda^{3,0}(M, I)$ the Dolbeault differential.

Proof. Theorem 3.7 (see also [GP], Proposition 2).

Definition 1.2. Let $M$ be a hypercomplex Hermitian manifold, $I, J, K \in \mathbb{H}$ the standard basis in quaternions, and

$$
\Omega \in \Lambda^{2,0}(M, I), \quad \Omega:=\frac{1}{2}\left(\omega_{J}+\sqrt{-1} \omega_{K}\right)
$$

\footnotetext{
${ }^{1}$ In other words, all elements of $V$ have weight 1 with respect to the $S U(2)$-action.
} 
the $(2,0)$-form constructed above. Then $M$ is called HKT-manifold (or hyperkähler with torsion) if

$$
\partial \Omega=0
$$

REMARK 1.3. By Lemma 1.1, the HKT-condition does not depend from the choice of a quaternion basis $I, J, K \in \mathbb{H}$.

The form $\Omega$ is clearly non-degenerate. The condition (1.1) means that $\Omega$ is "Dolbeault symplectic". This paper is an attempt to exploit the analogy between symplectic geometry and the HKT-geometry.

So far, almost every constructed example of a compact hypercomplex manifold comes bundled with a natural HKT-metrics. Moreover, there are no examples of a compact hypercomplex manifold where non-existence of an HKT-metrics is established. Therefore, HKT-manifolds are a natural object to study.

The most prominent example of an HKT-manifold is due to D. Joyce ([J]) and Spindel et al ([SSTV]) who constructed the hypercomplex structures on a compact Lie group, and Opfermann-Papadopoulos, who noticed that this manifold admits an HKT-metrics (see [OP], [GP]). Let $G$ be a compact semisimple Lie group. Then there exists a number $n$, depending on the geometry of a group $G$, such that the product of $G$ and an $n$-dimensional compact torus admits a left-invariant hypercomplex structure (Example 3.6). The HKT-metrics is provided by the Killing form.

1.3. The Kähler-de Rham superalgebra. In this paper, we study the Dolbeault complex of an HKT-manifold, from the viewpoint of Hodge theory and Kodaira relations. In Kähler geometry, the Kodaira relations might be interpreted as relations in a certain Lie superalgebra (see Subsection 4.1 for a definition of the Lie superalgebras). To be more precise, let $X$ be a Kähler manifold, $L, \Lambda, H$ the Lefschetz $S L(2)$ triple $([\mathrm{GH}])$, and $\partial, \bar{\partial}$ the Dolbeault differentials, considered as odd endomorphisms of the graded vector space $\Lambda^{*}(M)$. Consider the Lie superalgebra $\mathfrak{g} \subset \operatorname{End}\left(\Lambda^{*}(M)\right)$ generated by $L, \Lambda, H$ (even), $\partial$ and $\bar{\partial}$ (odd). The Kodaira relations are interpreted as the relations in the Lie superalgebra $\mathfrak{g}$ (see Section 4 ). From these relations it follows that the Lie algebra $\mathfrak{g}$ is independent from the choice of a Kähler manifold $X$. We call $\mathfrak{g}$ the Kähler-de Rham superalgebra.

1.4. The Kähler-de Rham superalgebra and HKT-geometry. Let $M$ be an HKT-manifold, $I, J, K \in \mathbb{H}$ the standard triple of induced complex structures, and $\left(\Lambda^{*, 0}(M, I), \partial\right)$ the Dolbeault differential graded (DG-) algebra of $(M, I)$. We use an analogy between $\left(\Lambda^{*, 0}(M, I), \partial\right)$ and the de Rham DG-algebra of a Kähler manifold. The role of the de Rham differential is played by the Dolbeault differential on $\Lambda^{*, 0}(M, I)$. The role of the Kähler form $\omega \in \Lambda^{1,1}(X)$ is played by the $(2,0)$ form $\Omega \in \Lambda^{2,0}(M, I)$ constructed above. One can associate with $\Omega$ an $S L(2)$-triple $L_{\Omega}, \Lambda_{\Omega}, H_{\Omega}$ acting on $\Lambda^{*, 0}(M, I)$ (Proposition 6.4).

We have the complex structure operator $I: \Lambda^{*}(X) \longrightarrow \Lambda^{*}(X)$ acting on the differential forms over a Kähler manifold $X$ in a multiplicative way. On the hypercomplex side, its role is played by an operator $\mathcal{J}$ defined as follows.

Let $J: \Lambda^{*}(M) \longrightarrow \Lambda^{*}(M)$ be the complex structure operator induced by $J \in$ $\mathbb{H}$. Since the quaternions $I$ and $J$ anti-commute, $J$ maps $\Lambda^{p, q}(M, I)$ to $\Lambda^{q, p}(M, I)$. 
Composing $J$ with the complex conjugation, we obtain an anticomplex automorphism

$$
\mathcal{J}: \Lambda^{p, q}(M, I) \longrightarrow \Lambda^{p, q}(M, I)
$$

We consider $\mathcal{J}$ as an endomorphism of $\Lambda^{*, 0}(M, I)$.

Twisting the Dolbeault differential with $\mathcal{J}$, we obtain a differential $\partial_{J}$, which is an analogue of the twisted de Rham differential $d^{c}$ on a Kähler manifold. Consider the Lie superalgebra generated by the Lefschetz triple $L_{\Omega}, \Lambda_{\Omega}, H_{\Omega}$ and the differentials $\partial, \partial_{J}$. We show that this Lie superalgebra is isomorphic to the Kähler-de Rham superalgebra (Corollary 7.2). In fact, this isomorphism is equivalent to the HKT-condition.

When the manifold $M$ is not only HKT, but also hyperkähler, the Dolbeault Laplacian $\partial \partial^{*}+\partial^{*} \partial$ is a central element of the Kähler-de Rham superalgebra constructed above. Therefore, we have a Lefschetz-type $S L(2)$-action on the Dolbeault cohomology $H^{*, 0}(M, I)$ of $(M, I)$. This is not very useful by itself, because $H^{*, 0}(M, I)$ of a compact hyperkähler manifold is very easy to compute. However, the same superalgebra acts on differential forms with coefficients in appropriate vector bundles (see [V1]). This is used to construct an $S L(2)$-action on the cohomology of these vector bundles. As an end result, we show that the deformation space of hyperholomorphic vector bundles is unobstructed, and prove that it is a hyperkähler variety (see [V1], [V2], [KV] for details).

A similar approach cannot work in whole generality for an HKT-manifold. Indeed, if we have a Lefschetz-type action on the Dolbeault cohomology of $(M, I)$, we have an isomorphism $H^{0}\left(\mathcal{O}_{(M, I)}\right) \cong H^{n}\left(\mathcal{O}_{(M, I)}\right)$, where $n=\operatorname{dim}_{\mathbb{C}} M$. By Serre's duality, $H^{n}\left(\mathcal{O}_{(M, I)}\right) \cong H^{0}(\mathrm{~K})^{*}$, where $\mathrm{K}:=K_{(M, I)}$ is the canonical bundle of $(M, I)$. This bundle is known to be non-trivial in some examples (for the Hopf surface, for example), and to have no non-trivial sections. But then, the 1-dimensional space $H^{0}\left(\mathcal{O}_{(M, I)}\right)$ would be isomorphic to the trivial space $H^{0}(\mathrm{~K})^{*}$, which is impossible. Therefore, one cannot expect an analogue of Lefschetz theorem to hold for the Dolbeault cohomology of $(M, I)$.

However, it is natural to expect some kind of Hodge theory to hold on the space $\Lambda^{*, 0}(M, I) \otimes \mathrm{K}^{1 / 2}$ of " $(p, 0)$ half-forms". First, by Serre's duality, we have

$$
H^{i}\left(\mathrm{~K}^{1 / 2}\right) \cong H^{n-i}\left(\mathrm{~K}^{1 / 2}\right)^{*}
$$

so the Lefschetz action identifies the spaces of the same dimension. Second, the space $\Lambda^{*, 0}(M, I) \otimes \mathrm{K}^{1 / 2}$ is naturally identified with the space of spinors on $M$, which is a Riemannian invariant of $M$. The corresponding cohomology space $H^{*}\left(\mathrm{~K}^{1 / 2}\right)$ is the space of harmonic spinors, and it is also a Riemannian invariant. Thus, the half-forms and the cohomology of $\mathrm{K}^{1 / 2}$ are in some sense more relevant geometrically than the Dolbeault cohomology.

This paper is dedicated to revealing the Lefschetz-type action on the harmonic spinors.

First of all, we notice that the canonical bundle $\mathrm{K}$ of $(M, I)$ is topologically trivial. Indeed, taking the top exterior power of the symplectic form $\Omega \in \Lambda^{2,0}(M, I)$, we obtain a nowhere degenerate section of $K$. Using this trivialization, one defines the square root of $\mathrm{K}$ in a usual way (see Subsection 10.2). The endomorphism

$$
\mathcal{J}: \Lambda^{p, 0}(M, I) \otimes \mathrm{K}^{1 / 2} \longrightarrow \Lambda^{p, 0}(M, I) \otimes \mathrm{K}^{1 / 2}
$$


is defined in the same fashion as above. Let ${ }^{n} \partial$ be the Dolbeault differential in $\Lambda^{*, 0}(M, I) \otimes \mathrm{K}^{1 / 2}$,

$$
{ }^{n} \partial: \Lambda^{p, 0}(M, I) \otimes \mathrm{K}^{1 / 2} \longrightarrow \Lambda^{p+1,0}(M, I) \otimes \mathrm{K}^{1 / 2},
$$

and ${ }^{n} \partial_{J}$ the twisted differential,

$$
{ }^{n} \partial_{J}:=-\mathcal{J} \circ{ }^{n} \partial \circ \mathcal{J} .
$$

The Lefschetz $S L(2)$-triple $L_{\Omega}, \Lambda_{\Omega}, H_{\Omega}$ acts on $\Lambda^{*, 0}(M, I) \otimes \mathrm{K}^{1 / 2}$ in a natural way. The main result of this paper is the following

Theorem 1.4. [ Subsection 10.1 ] Let $M$ be a HKT-manifold, $I, J, K \in \mathbb{H}$ the standard basis in quaternions, and $\mathrm{K}^{1 / 2}$ the square root of the canonical bundle of $(M, I)$, constructed above. Consider the Lefschetz $S L(2)$-triple $L_{\Omega}, \Lambda_{\Omega}, H_{\Omega}$ acting on the bundle of $(p, 0)$-half-forms $\Lambda^{*, 0}(M, I) \otimes \mathrm{K}^{1 / 2}$ defined above. Let

$$
{ }^{n} \partial: \Lambda^{p, 0}(M, I) \otimes \mathrm{K}^{1 / 2} \longrightarrow \Lambda^{p+1,0}(M, I) \otimes \mathrm{K}^{1 / 2},
$$

be the Dolbeault operator, and ${ }^{n} \partial_{J}$ the twisted differential,

$$
{ }^{n} \partial_{J}:=-\mathcal{J} \circ{ }^{n} \partial \circ \mathcal{J} \text {. }
$$

Consider the Lie superalgebra $\mathfrak{g}$ generated by the even operators $L_{\Omega}, \Lambda_{\Omega}, H_{\Omega}$ and the odd operators ${ }^{n} \partial,{ }^{n} \partial_{J}$, Then $\mathfrak{g}$ is isomorphic to the Kähler-de Rham superalgebra.

Proof. See Subsection 10.1.

COROLLARY 1.5. We have a Lefschetz-type $S L(2)$-action on the cohomology $H^{*}\left(\mathrm{~K}^{1 / 2}\right)$.

Proof. This is Theorem 10.2.

This result should be especially useful when the canonical bundle $\mathrm{K}$ of $(M, I)$ is trivial. However, we have not found examples of compact HKT- (or even hypercomplex) manifolds which are not hyperkähler and have trivial canonical bundle. Moreover, in all non-hyperkähler examples where we have computed the group $H^{i}\left(\mathrm{~K}^{1 / 2}\right)$, this group was trivial, for all $i$. Still, the action of the Kähler-de Rham superalgebra on the space of spinors is quite remarkable.

1.5. Non-Kähler manifolds and Calabi-Yau geometry. As we have seen above, the canonical bundle of a hypercomplex manifold is topologically trivial. Therefore, these manifolds may be regarded as "non-Kähler Calabi-Yau manifolds". The study of such manifolds is quite important, due to the following conjecture of M. Reid. Let $X$ be a 3-dimensional Calabi-Yau manifold. Suppose that $X$ contains a rational curve which can be blown down; denote the blow-down of $X$ by $\widetilde{X}_{1}$. Consider a generic small deformation $X_{1}$ of $\widetilde{X}_{1}$. It is known that all singularities of 3-dimensional Calabi-Yau manifolds can be removed by a small deformation. Therefore, we may assume that $X_{1}$ is a smooth manifold, which has trivial canonical class and satisfies $h^{2}\left(X_{1}\right)=h^{2}(X)-1$. Repeating this process, we obtain a manifold $X_{n}$ with $h^{2}\left(X_{n}\right)=0$. This manifold is, of course, non-Kähler. However, its topological 
structure is very simple. Namely, $X_{n}$ is diffeomorphic to a connected sum $\#{ }_{k} S^{3} \times S^{3}$ of $k$ copies of a $S^{3} \times S^{3}$, where $S^{3}$ is a 3-dimensional sphere, and $k=h^{3}(X) / 2$.

M. Reid conjectured that, starting from another 3-dimensional Calabi-Yau manifold $Y, h^{3}(Y)=h^{3}(X)$, we obtain a non-Kähler manifold $Y_{n^{\prime}}$ in the same deformation class as $X_{n}$,

This conjecture is significant because, in Calabi-Yau geometry, most results deal with complete intersections in toric and homogeneous manifolds, and the general Calabi-Yau manifolds are quite difficult to study. The M. Reid's conjecture gives a possibility to reduce a given Calabi-Yau manifold to a toric Calabi-Yau by a series of birational transforms and deformations. This way, one might hope to extend the standard results about complete intersections in toric manifolds (the Mirror Conjecture, for instance) to the general case.

The M. Reid's conjecture is difficult to tackle because very little is known about the geometry of non-Kähler Calabi-Yau manifolds. The present paper can be read as an attempt to study their geometry, from the Hodge-theoretic point of view. The analogy is straightforward. The main working example of an HKT manifold, the compact group $S U(3)$, looks very similar, from the topological and geometrical point of view, to the manifold $\#_{k} S^{3} \times S^{3}$. The simplest 3-dimensional "non-Kähler CalabiYau" manifold $S^{3} \times S^{3}$ is actually a Lie group; and the Lie group $S^{3} \times S^{1}$ is also an HKT-manifold, called the Hopf surface.

\subsection{Contents.}

- This Introduction (Section 1) is independent from the rest of this paper.

- In Section 2, we recite some basic results and conventions of hypercomplex and hyperkaehler geometry. This material is standard (see, e.g., [KV]).

- In Section 3, we repeat the definition and some basic properties of HKTmanifolds ([HP], [GP]).

- In Section 4, we study the natural superalgebra of a Kähler manifold ([FKS]).

- In Section 5, we define and study the quaternionic Dolbeault complex, following S. Salamon (see [CS] and [V3]).

- In Section 6, we study the supersymmetry of a hyperkähler manifold, following $[\mathrm{FKS}]$ and $[\mathrm{V} 0]$.

- In Section 7, we prove that the Dolbeault algebra of an HKT-manifold admits the same kind of supersymmetry as the de Rham algebra of a Kähler manifold.

- In Sections 8-9, we obtain some commutation relations in the superalgebra of the Dolbeault complex of an HKT-manifold.

- In Section 10, we apply these commutation relation to establish the geometrically relevant kind of supersymmetry on the bundle of spinors. This is used to show that the harmonic spinors admit a natural Lefschetz-type $S L(2)$-action

\section{Hypercomplex manifolds.}

2.1. Hypercomplex Hermitian manifolds. Definition 2.1. Let $M$ be a smooth manifold equipped with an action of the quaternion algebra $\mathbb{H}$ in its tangent space. Assume that for all quaternions $L \in \mathbb{H}, L^{2}=-1$, the almost complex structure given by $L \in \operatorname{End}(T M)$ is integrable. Then $M$ is called hypercomplex.

REMARK 2.2. By D. Kaledin's theorem ([K]), in Definition 2.1 it suffices to check integrability only for $L_{1}, L_{2} \in \mathbb{H}, L_{1} \neq \pm L_{2}$. The integrability of $L_{1}, L_{2}$ implies integrability of any almost complex structure given by $L \in \mathbb{H}, L^{2}=-1$. 
This paper is dedicated to the study of hypercomplex manifolds. For this purpose, we introduce a natural kind of Riemannian metrics, called quaternionic Hermitian. It is related to the hypercomplex structure in the same way as the usual Hermitian metrics on a complex manifold is related to a complex structure. This definition is purely linear-algebraic.

Let $V$ be a quaternionic vector space. Given $L \in \mathbb{H}, L^{2}=-1, L$ defines a complex structure on $V$. We denote $V$, considered as a complex vector space, by $(V, L)$. The following lemma is trivial.

LEMMA 2.3. Let $V$ be a quaternionic vector space, and $h: V \times V \longrightarrow \mathbb{R}$ a positive bilinear symmetric form. Then the following conditions are equivalent.

(i) For any $L \in \mathbb{H}, L^{2}=-1$, the metrics $h$ is Hermitian on the complex vector space $(V, L)$.

(ii) Consider the action of the group $S U(2)$ of unitary quaternions on $V$. Then $h$ is $S U(2)$-invariant.

Proof. Clear.

DEFINITION 2.4. Let $V$ be a quaternionic vector space, and $h: V \times V \rightarrow \mathbb{R}$ a positive bilinear symmetric form. Then space $(V, h)$ is called a quaternionic Hermitian space if any of the conditions of Lemma 2.3 holds. In this case, the metrics $h$ is also called quaternionic Hermitian.

Definition 2.5. Let $M$ be a hypercomplex manifold, and $s$ a Riemannian structure on $M$. We say that $M$ is hypercomplex Hermitian if for all $x \in M$, the tangent space $\left(T_{x} M, s\right)$ is quaternionic Hermitian.

Definition 2.6. Let $M$ be a hypercomplex manifold, $L \in \mathbb{H}, L^{2}=-1$. By definition, $L$ induces a complex structure on $M$. Thus obtained complex manifold is denoted by $(M, L)$. The complex structure $L$ is called induced by the hypercomplex structure. If, in addition, $M$ is hypercomplex Hermitian, then $(M, L)$ is a complex Hermitian manifold. The corresponding real-valued skew-symmetric 2-form is denoted by $\omega_{L} \in \Lambda^{1,1}(M, L)$,

$$
\omega_{L}(x, y)=s(x, L y)
$$

where $s$ denotes the Riemannian form.

Definition 2.7. Let $M$ be a hypercomplex Hermitian manifold. Then $M$ is called hyperkähler if for any induced complex structure $L \in \mathbb{H}, L^{2}=-1$, the corresponding Hermitian manifold $(M, L)$ is Kähler.

Let $M$ be a hypercomplex Hermitian manifold, and $I, J, K \in \mathbb{H}$ the standard triple of quaternions. Consider the 2-form

$$
\Omega:=\frac{1}{2}\left(\omega_{J}+\sqrt{-1} \omega_{K}\right)
$$

An elementary linear-algebraic computation shows that $\Omega$ is of type $(2,0)$ with over $(M, I)$. 
Claim 2.8. Let $M$ be a hypercomplex Hermitian manifold, $I, J, K \in \mathbb{H}$ the standard triple of quaternions, and $\Omega \in \Lambda^{2,0}(M, I)$ the $(2,0)$-form constructed above. Then $M$ is hyperkähler if and only if $d \Omega=0$.

Proof. The proof is well known (see [Bes]).

2.2. The natural $S U(2)$-action on the differential forms. Let $M$ be a hypercomplex manifold. We identify the group $S U(2)$ with the group of unitary quaternions. This gives a canonical action of $S U(2)$ on the tangent bundle, and all its tensor powers. In particular, we obtain a natural action of $S U(2)$ on the bundle of differential forms.

The corresponding Lie algebra action is related to the usual Hodge decomposition as follows. Let $L$ be an induced complex structure, and

$$
\Lambda^{*}(M)=\oplus \Lambda^{p, q}(M, L)
$$

be the associated Hodge decomposition. Consider an operator

$$
\operatorname{adL}: \Lambda^{i}(M) \longrightarrow \Lambda^{i}(M)
$$

acting as $\eta \longrightarrow(p-q) \sqrt{-1} \eta$ on $\eta \in \Lambda^{p, q}(M, L)$.

Lemma 2.9. Let $M$ be a hypercomplex manifold, $I, J, K \in \mathbb{H}$ the standard triple of quaternions, and $a d I, a d J, a d K$ the corresponding endomorphisms of $\Lambda^{*}(M)$. Then $a d I$, adJ, adK generate the Lie algebra $\mathfrak{s u}(2) \subset \operatorname{End}\left(\Lambda^{*}(M)\right)$ associated with the hypercomplex structure.

Proof. Clear (see e.g. [V0], [V1]).

\section{HKT-manifolds.}

3.1. Busmut connections. For a reference and a bibliography on Bismut connections, see [GP].

Let $M$ be a complex manifold equipped with a Hermitian metrics, and $\nabla$ a connection (not necessarily torsion-free) on $T M$ which preserves the Hermitian metrics and the complex structure. Denote its torsion by $T_{\nabla} \in \Lambda^{2} T^{*} M \otimes T M$. Using the Riemannian structure to identify $T M$ and $T^{*} M$, we may consider $T_{\nabla}$ as an element of $\Lambda^{2} M \otimes \Lambda^{1} M \subset\left(T^{*} M\right)^{\otimes 3}$.

Definition 3.1. The connection $\nabla$ is called Bismut connection if the tensor

$$
T_{\nabla} \in \Lambda^{2} M \otimes \Lambda^{1} M \subset\left(T^{*} M\right)^{\otimes 3}
$$

is totally skew-symmetric, that is, belongs to the space of differential forms $\Lambda^{3}(M) \subset$ $\left(T^{*} M\right)^{\otimes 3}$.

Theorem 3.2. (Chern) Let $M$ be a complex Hermitian manifold. Then the Bismut connection exists and is unique.

Proof. Well known ([GP]). The proof is constructed along the same lines as the proof of the existence and uniqueness of a Levi-Civita connection on a Riemannian 
manifold. The space of connections is affine; one considers the map $\nabla \longrightarrow T_{\nabla}$ as an affine map of affine spaces, and estimates its kernel and cokernel by dimension count. 口

REMARK 3.3. Let $M$ be a complex Hermitian manifold, $\nabla$ the Bismut connection, and $T_{\nabla} \in \Lambda^{3}(M)$ the torsion tensor, considered as a differential form as in Definition 3.1. It is possible to express $T_{\nabla}$ in terms of the standard skew-symmetric form $\omega \in \Lambda^{1,1}(M)$ associated with the Hermitian form. Namely, let $I: \Lambda^{3}(M) \longrightarrow \Lambda^{3}(M)$ be the complex structure operator extended to $\Lambda^{3}(M)$ by multiplicativity. Then $T_{\nabla}=I(d \omega)([\mathrm{GP}])$.

3.2. HKT-manifolds: the definition. HKT-manifolds were introduced by P.S.Howe and G.Papadopoulos ([HP]). For a reference and a bibliography on HKTmanifolds, see [GP].

Definition 3.4. [HP] Let $M$ be a hypercomplex Hermitian manifold (Definition 2.5). For an induced complex structure $L=I, J, K$, consider the corresponding complex Hermitian manifold $(M, L)$. Let $\nabla_{L}$ be the associated Bismut connection (Theorem 3.2). We say that $M$ is HKT- (hyperkähler with torsion) manifold if

$$
\nabla_{I}=\nabla_{J}=\nabla_{K}
$$

REMARK 3.5. An HKT-manifold is not hyperkähler. Therefore, the term "hyperkähler with torsion" is not legitimate and actually misleading. This is why we, throughout this paper, prefer to use the term "HKT-manifold" instead.

EXAMPLE 3.6. Let $G$ be a compact Lie group from the following list:

$$
\begin{aligned}
& S U(2 l+1), T^{1} \times S U(2 l), \quad T^{l} \times S O(2 l+1), \quad T^{l} \times S p(l), \\
& T^{2 l-1} \times S O(4 l+2), \quad T^{2} \times E_{6}, \quad T^{7} \times E_{7}, \\
& T^{4} \times F_{4}, \quad T^{2} \times G_{2} .
\end{aligned}
$$

where $T^{i}=\left(S^{1}\right)^{i}$ is an $i$-dimensional compact torus. D. Joyce ([J]) and independently Spindel et al. ([SSTV]) have shown that $G$ is equipped with a family of natural left-invariant hypercomplex structures. The corresponding complex structures were constructed in 1950-ies by Bott and Samelson ([Sa]).

Consider the Killing metrics $(\cdot, \cdot)$ on $G$. Then $(\cdot, \cdot)$ is hypercomplex Hermitian, with respect to the hypercomplex structure obtained by D. Joyce. Consider $G$ as a hypercomplex Hermitian manifold. Then $G$ is an HKT-manifold ([OP]).

Denote the unit of $G$ by $e$. The corresponting Lie algebra $\mathfrak{g}$ is identified with $T_{e} G$. Denote the structure constants of $\mathfrak{g}$ by

$$
C_{b, c}^{a}: T_{e} G \times T_{e} G \longrightarrow T_{e} G
$$

Using the Killing form, we may identify $T_{e} G$ and $T_{e}^{*} G$. Using this identification, we consider $C_{b, c}^{a}$ as a 3 -form $\mathcal{T}: T_{e} G \times T_{e} G \times T_{e} G \longrightarrow \mathbb{R}$. It is well known that $\mathcal{T}$ is totally antisymmetric. An exterior form on $T_{e} G$ can be uniquely extended to a left invariant differential form on $G$. Denote by $\mathfrak{T}$ the differential form obtained from $\mathcal{T}$ this way. Then $\mathfrak{T}$ is the torsion form of the HKT-connection $\nabla$ on $G$ (Definition 3.4). 
Moreover, $\nabla$ is the standard left-linvariant torsion connection on the group $G$; a vector field $v$ is parallel with respect to $\nabla$ if and only if $v$ is left-invariant.

Further details on this can be found in [GP].

The definition of an HKT-manifold is somewhat unwieldy, as it uses the "black box" of the existence and uniqueness of Bismut connection (Theorem 3.2). It is better to use the following theorem instead, which is a version of a result from [HP].

Theorem 3.7. ([GP], Proposition 2) Let $M$ be a quaternionic Kähler manifold. Given a triple of induced complex structures

$$
I, J, K, \quad I \circ J=-J \circ I=K
$$

consider the corresponding non-degenerate (2,0)-form $\Omega \in \Lambda_{I}^{2,0}(M)(2.1)$. Let $\partial$ : $\Lambda_{I}^{2,0}(M) \longrightarrow \Lambda_{I}^{3,0}(M)$ be the Dolbeault differential, and $d$ the de Rham differential. Then the following conditions are equivalent

(i) $\partial \Omega=0$.

(ii) Let $\omega_{I}, \omega_{J}, \omega_{K}$ be the standard 2-forms on $M(2.1)$. The 3 -forms $d \omega_{I}, d \omega_{J}, d \omega_{K}$ all have weight 1 with respect to the natural $S U(2)$-action on $\Lambda^{3}(M){ }^{1}$

(iii) $M$ is an HKT-manifold.

Proof. The equivalence (i) $\Leftrightarrow$ (iii) is proven in [GP], Proposition 2. The implication (ii) $\Rightarrow$ (i) is clear, because $\partial \Omega$ is a linear combination of the 3 -forms $d \omega_{J}, d \omega_{K}$, $d^{c} \omega_{J}, d^{c} \omega_{K}$ (by $d^{c}$ we denote the "twisted de Rham differential", $d^{c}:=-I \circ d \circ I$ ). These four 3-forms all belong to the subrepresentation of $\Lambda^{3}(M)$ generated by $d \omega_{J}$, $d \omega_{K}$, hence by (ii) they have weight 1 with respect to $S U(2)$. On the other hand, by Lemma 2.9 , a $(3,0)$-form $\partial \Omega \in \Lambda_{I}^{3,0}(M)$ has weight 3 if it is non-zero. Finally, an implication (iii) $\Rightarrow$ (ii) follows directly from the Corollary 1 of [GP] (see also [HP]).

One can easily deduce the proof of Theorem 3.7 using the quaternionic Dolbeault complex (see Remark 5.9).

REMARK 3.8. The standard $S U(2)$-action on $\Lambda^{3}(M)$ has weights 3 and 1 . It is easy to see that the weight 3 component is generated by $\Lambda_{I}^{3,0}(M)$ for all induced complex structures $I \in \mathbb{H}$. This explains the implication Theorem 3.7 (i) $\Rightarrow$ Theorem 3.7 (ii).

EXAMPLE 3.9. Let $M$ be a hypercomplex Hermitian manifold, of real dimension 4 . Since $\Lambda^{3}(M) \cong \Lambda^{1}(M)$, the group $S U(2)$ acts on $\Lambda^{3}(M)$ with weight 1 . By Theorem 3.7, then, $M$ is a HKT-manifold.

4. The Kähler-de Rham superalgebra. In this Section, we give a novel presentation of Kodaira relations, which are interpreted as relations in a certain Lie superalgebra. These ideas are commonplace in physics; for a physical interpretations of the Lie superalgebra generated by de Rham and Dolbeault differentials and the Hodge operators, see [FKS].

\footnotetext{
${ }^{1}$ This means that each of these forms belongs to a weight $1 S U(2)$-subspace of $\Lambda^{3}(M)$. An $S U$ (2)-representation is of weight 1 if it is a direct sum of several copies of a standard 2-dimensional irreducible representation.
} 
4.1. Lie superalgebras. Let $A$ be a $\mathbb{Z} / 2 \mathbb{Z}$-graded vector space,

$$
A=A^{\text {even }} \oplus A^{\text {odd }} \text {. }
$$

We say that $a \in A$ is pure if $a$ belongs to $A^{\text {even }}$ or $A^{\text {odd }}$. For a pure element $a \in A$, we write $\widetilde{a}=0$ if $a \in A^{\text {even }}$, and $\widetilde{a}=1$ if $a \in A^{\text {odd }}$. Consider a bilinear operator

$$
[\cdot, \cdot]: A \times A \longrightarrow A,
$$

called supercommutator. Assume that $[\cdot, \cdot]$ is graded anti-commutative, that is, satisfies

$$
[a, b]=-(-1)^{\tilde{a} \tilde{b}}[b, a]
$$

for pure $a, b \in A$. Assume, moreover, that $[\cdot, \cdot]$ is compatible with the grading: the commutator $[a, b]$ is even when both $a, b$ are even or odd, and odd if one of these elements is odd and another is even. We say that $A,[\cdot, \cdot]$ is a Lie superalgebra, if the following identity (called the graded Jacoby identity) holds, for all pure elements $a, b, c \in A$

$$
[a,[b, c]]=[[a, b], c]+(-1)^{\tilde{a} \tilde{b}}[b,[a, c]] .
$$

Up to a sign, this is a usual Jacoby identity.

Every reasonable property of Lie algebras has a natural analogue for Lie superalgebras; the rule of thumb, every time one would exchange two elements $a$ and $b$, one adds a multiplier $(-1)^{\tilde{a} \tilde{b}}$.

In classical terms, one interprets the Lie superalgebras as follows: $A^{\text {even }}$ is a usual Lie algebra, $A^{\text {odd }}$ is an $A^{\text {even }}$-module, equipped with a bilinear symmetric $A^{\text {even }}$ invariant pairing

$$
[\cdot, \cdot]: A^{\text {odd }} \times A^{\text {odd }} \longrightarrow A^{\text {even }}
$$

which satisfies the (super-)Jacoby identity

$$
[a,[b, c]]=[[a, b], c]+[[a, c], b]
$$

for all $a, b, c \in A^{\text {odd }}$.

EXAMPLE 4.1. Let $V=V^{\text {even }} \oplus V^{\text {odd }}$ be a $\mathbb{Z} / 2 \mathbb{Z}$-graded vector space, and End $V$ its endomorphism space, equipped with induced grading. We define a supercommutator in End $V$ by the formula

$$
[a, b]=a b-(-1)^{\tilde{a} \tilde{b}} b a
$$

It is easy to check that (End $V,[\cdot, \cdot]$ ) is a Lie superalgebra.

4.2. Kodaira relations and the Kähler-de Rham superalgebra. Let $M$ be a Kähler manifold. Consider $\Lambda^{*}(M)$ as a graded vector space. The differentials $d, d^{c}:=-I \circ d \circ I$ can be interpreted as odd elements in $\operatorname{End}\left(\Lambda^{*}(M)\right)$, and the Hodge operators $L, \Lambda, H$ as even elements. To avoid confusion, we denote the supercommutator of odd elements as $\{\cdot, \cdot\}$. In terms of the associative algebra, $\{a, b\}=a b+b a$. Let $d^{*}:=\left[\Lambda, d^{c}\right],\left(d^{c}\right)^{*}:=-[\Lambda, d]$. The usual Kodaira relations can be stated as follows 


$$
\begin{aligned}
& {\left[L, d^{*}\right]=-d^{c}, \quad\left[L,\left(d^{c}\right)^{*}\right]=d, \quad\left\{d,\left(d^{c}\right)^{*}\right\}=\left\{d^{*}, d^{c}\right\}=0} \\
& \left\{d, d^{c}\right\}=\left\{d^{*},\left(d^{c}\right)^{*}\right\}=0, \quad\left\{d, d^{*}\right\}=\left\{d^{c},\left(d^{c}\right)^{*}\right\}=\Delta
\end{aligned}
$$

where $\Delta$ is the Laplace operator, commuting with $L, \Lambda, H$, and $d, d^{c}$.

DEFINITION 4.2. Let $M$ be a Kähler manifold. Consider the Lie superalgebra $\mathfrak{g} \subset \operatorname{End}\left(\Lambda^{*}(M)\right)$ generated by the even elements $L, \Lambda, H$, and the odd elements $d, d^{c}$ Then $\mathfrak{g}$ is called the Kähler-de Rham superalgebra associated with $M$. $4.3)$.

Using (4.2), is easy to see that $\mathfrak{g}$ is in fact independent form $M$ (see Subsection

This Lie superalgebra was studied from the physicists' point of view in [FKS].

4.3. Axiomatics of the Kähler-de Rham superalgebra. The Kähler-de Rham superalgebra has the following formal interpretation.

ThEOREM 4.3. Let $\mathfrak{g}$ be a Lie superalgebra, and $\mathfrak{a} \subset \mathfrak{g}^{\text {even }}$ its even subalgebra isomorphic to $\mathfrak{s l}(2)$, and generated by the standard $\mathfrak{s l}(2)$-triple $\langle L, \Lambda, H\rangle \in \mathfrak{g}^{\text {even }}$. Consider odd vectors $d, d^{c} \subset \mathfrak{g}^{\text {odd }}$. Assume that $[H, d]=d,\left[H, d^{c}\right]=d^{c}$, and $[L, d]=$ $\left[L, d^{c}\right]=0$, so that $d$ and $d^{c}$ generate weight-1-representations of $\mathfrak{a} \cong \mathfrak{s l}(2)$. Assume, moreover, that $\mathfrak{g}$ is generated as a superalgebra by $\left(L, \Lambda, H, d, d^{c}\right)$ Finally, assume that

$$
\{d, d\}=\left\{d^{c}, d^{c}\right\}=\left\{d, d^{c}\right\}=0,
$$

where $\{\cdot, \cdot\}$ denotes the super-commutator of odd elements. Consider the vectors

$$
d^{*}:=\left[\Lambda, d^{c}\right], \quad\left(d^{c}\right)^{*}:=-[\Lambda, d] .
$$

Then

(i) We have

$$
\left\{d, d^{*}\right\}=\left\{d^{c},\left(d^{c}\right)^{*}\right\}
$$

Denote this supercommutator by

$$
\Delta:=\left\{d, d^{*}\right\}=\left\{d^{c},\left(d^{c}\right)^{*}\right\} .
$$

(ii) The Lie superalgebra $\mathfrak{g}$ is 8 -dimensional, and spanned by

$$
L, \Lambda, H, d, d^{c}, d^{*},\left(d^{c}\right)^{*}, \Delta .
$$

Moreover, $\mathfrak{g}$ is naturally isomorphic to the Kähler-de Rham superalgebra (Definition 4.2).

Proof. The action of the $\mathfrak{s l}(2)$-triple $\langle L, \Lambda, H\rangle$ on

$$
V:=\left\langle d, d^{c}, d^{*},\left(d^{c}\right)^{*}\right\rangle \subset \mathfrak{g}
$$

is transparent, because $V$ is a direct sum of two weight 1 representations, $\left\langle d,\left(d^{c}\right)^{*}\right\rangle$ and $\left\langle d^{c}, d^{*}\right\rangle$. To prove Theorem 4.3 , we have to show that the commutator relations 
in the 4-dimensional odd space $\left\langle d, d^{c}, d^{*},\left(d^{c}\right)^{*}\right\rangle \subset \mathfrak{g}$ are consistent with (4.2), and to show that $\Delta$ belongs to the center of $\mathfrak{g}$.

We start with proving (4.4). Consider the relation

$$
\left\{d, d^{c}\right\}=0
$$

(see (4.3)). Since the super-commutator in $V$ is a-invariant, (4.5) implies

$$
\left[\Lambda,\left\{d, d^{c}\right\}\right]=\left\{[\Lambda, d], d^{c}\right\}+\left\{d,\left[\Lambda, d^{c}\right]\right\}=0
$$

which means

$$
\left\{\left(d^{c}\right)^{*}, d^{c}\right\}-\left\{d, d^{*}\right\}=0 .
$$

This proves Theorem 4.3 (i). Remaining commutation relations between $d, d^{c}, d^{*},\left(d^{c}\right)^{*}$ are obtained in a similar way, as follows.

Acting on $\{d, d\}=0$ with $[\Lambda, \cdot]$, we obtain

$$
\{[\Lambda, d], d\}=0
$$

that is, $\left\{\left(d^{c}\right)^{*}, d\right\}=0$. Similarly, $\left\{d^{c}, d^{c}\right\}=0$ acted on by $[\Lambda, \cdot]$ implies $\left\{d^{*}, d^{c}\right\}=0$. Since the vectors $d, d^{c}$ have weight 1 with respect to the $\mathfrak{s l}(2)$-action $L, \Lambda, H$, we have

$$
[\Lambda,[\Lambda, d]]=\left[\Lambda,\left[\Lambda, d^{c}\right]\right]=0 .
$$

In other words,

$$
\left[\Lambda, d^{*}\right]=\left[\Lambda,\left(d^{c}\right)^{*}\right]=0 .
$$

Acting with $[\Lambda, \cdot]$ on both sides of $\left\{d^{*}, d^{c}\right\}=0$, and using (4.6), we obtain

$$
0=\left[\Lambda,\left\{d^{*}, d^{c}\right\}\right]=\left\{\left[\Lambda, d^{*}\right], d^{c}\right\}+\left\{d^{*},\left[\Lambda, d^{c}\right]\right\}=\left\{d^{*},\left[\Lambda, d^{c}\right]\right\}=\left\{d^{*}, d^{*}\right\}
$$

Using $\left\{\left(d^{c}\right)^{*}, d\right\}=0$ instead of $\left\{d^{*}, d^{c}\right\}=0$ and applying the same argument, obtain $\left\{\left(d^{c}\right)^{*},\left(d^{c}\right)^{*}\right\}=0$. Finally, if we apply $[\Lambda, \cdot]$ to

$$
\left\{\left(d^{c}\right)^{*}, d^{c}\right\}-\left\{d, d^{*}\right\}=0,
$$

we shall have

$$
\left\{\left(d^{c}\right)^{*}, d^{*}\right\}+\left\{\left(d^{c}\right)^{*}, d^{*}\right\}=0
$$

that is,

$$
\left\{\left(d^{c}\right)^{*}, d^{*}\right\}=0
$$

We have found that pairwise super-commutators of the vectors $d, d^{c}, d^{*},\left(d^{c}\right)^{*}$ are equal zero, except

$$
\left\{d, d^{*}\right\}=\left\{d^{c},\left(d^{c}\right)^{*}\right\}=\Delta .
$$

We obtained the Kodaira identities (4.2). To prove Theorem 4.3, it remains to show that the "Laplacian" $\Delta$ commutes with

$$
d, d^{c}, L, \Lambda, H
$$


Writing $\Delta$ as $\left\{d, d^{*}\right\}$ and using the commutation relations between $d^{c},\left(d^{c}\right)^{*}$ and $d, d^{*}$, we find that $\Delta$ commutes with $d^{c}$ and $\left(d^{c}\right)^{*}$. Similarly, using the formula $\Delta=\left\{d^{c},\left(d^{c}\right)^{*}\right\}$ and the fact that $d, d^{*}$ commutes with $d^{c},\left(d^{c}\right)^{*}$, we find that $d, d^{*}$ commutes with $\Delta$. The operator $H$ commutes with $\Delta$ because $H$ multiplies $d$ by 1 and $d^{*}$ by -1 . Finally, $L$ and $\Lambda$ commute with $\Delta$ because $L$ maps $d$ to zero and $d^{*}$ to $d^{c}$, hence

$$
\left[L,\left\{d, d^{*}\right\}\right]=\left\{[L, d], d^{*}\right\}+\left\{d,\left[L, d^{*}\right]\right\}=\left\{d, d^{c}\right\}
$$

this commutator is equal zero by (4.5). Similarly,

$$
\left[\Lambda,\left\{d, d^{*}\right\}\right]=\left\{[\Lambda, d], d^{*}\right\}+\left\{d,\left[\Lambda, d^{*}\right]\right\}=-\left\{d^{*},\left(d^{c}\right)^{*}\right\}
$$

this is equal zero by (4.7). We have proven Theorem 4.3.

4.4. Kähler-de Rham superalgebra and the odd Heisenberg superalgebra. DEFINITION 4.4. Let $\mathfrak{g}$ be the Lie superalgebra defined by generators and relations as in Theorem 4.3. Then $\mathfrak{g}$ is called the Kähler-de Rham superalgebra.

The Kähler-de Rham superalgebra can be described explicitly as follows. Let $V$ be a weight 1 representation of $\mathfrak{s l}(2)$. Consider the space $V \oplus V^{*}$ with the natural $\mathfrak{s l}(2)$ invariant bilinear symmetric form $\langle\cdot, \cdot$,$\rangle . Consider the graded space \mathfrak{h}:=V \oplus V^{*} \oplus \mathbb{C} c$, with $\mathbb{C} c 1$-dimensional even space and $V \oplus V^{*}$ odd. We define a supercommutator on $\mathfrak{h}$ in such a way that

$$
\left\{v_{1}, v_{2}\right\}:=\left\langle v_{1}, v_{2}\right\rangle c, \text { for all } v_{1}, v_{2} \in V \oplus V^{*}
$$

and $c$ is a central element. The Lie superalgebra $\mathfrak{h}$ is called odd Heisenberg algebra associated with $V \oplus V^{*}$. By construction, $\mathfrak{s l}(2)$ acts in $\mathfrak{h}$ by Lie superalgebra automorphisms.

Proposition 4.5. In the above notation, consider the semidirect product $\mathfrak{s l}(2) 人$ $\mathfrak{h}$. Then $\mathfrak{s l}(2) \wedge \mathfrak{h}$ is isomorphic to the Kähler-de Rham Lie superalgebra $\mathfrak{g}$.

Proof. Consider the odd 4-dimensional subspace $W \subset \mathfrak{g}$ spanned by $d, d^{c}, d^{*}$, $\left(d^{c}\right)^{*}$. By Kodaira idenitities, the commutator of vectors $v, v^{\prime} \in W$ is proportional to the Laplacian. Let

$$
\left\langle v, v^{\prime}\right\rangle:=\frac{\left\{v, v^{\prime}\right\}}{\Delta}
$$

Clearly, $W$ generates a 5-dimensional subalgebra $\mathfrak{k} \subset \mathfrak{g}$ which is naturally isomorphic to $\mathfrak{h}$. The Hodge operators $L, \Lambda, H$ commute with the Laplacian, and therefore preserve the bilinear form $\left\langle v, v^{\prime}\right\rangle$. Therefore, the $\mathfrak{s l}(2)$-algebra generated by $L, \Lambda, H$ acts on $\mathfrak{k}$ in the same way as $\mathfrak{s l}(2)$ acts on $\mathfrak{h}$. Finally, $\mathfrak{g}$ is by construction a semidirect product of $\mathfrak{s l}(2)$ and $\mathfrak{k}$.

5. Quaternionic Dolbeault complex. The quaternionic cohomology is a well known subject, introduced by M. Capria and S. Salamon ([CS]). Here we give an exposition of quaternionic cohomology and quaternionic Dolbeault complex for hypercomplex manifolds. We follow [V3]. 
5.1. Quaternionic Dolbeault complex: the definition. Let $M$ be a hypercomplex manifold, and

$$
\Lambda^{0} M \stackrel{d}{\longrightarrow} \Lambda^{1} M \stackrel{d}{\longrightarrow} \Lambda^{2} M \stackrel{d}{\longrightarrow} \ldots
$$

its de Rham complex. Consider the natural action of $S U(2)$ on $\Lambda^{*} M$. Clearly, $S U(2)$ acts on $\Lambda^{i} M, i \leqslant \frac{1}{2} \operatorname{dim}_{\mathbb{R}} M$ with weights $i, i-2, i-4, \ldots$

We denote by $\Lambda_{+}^{i}$ the maximal $S U(2)$-subspace of $\Lambda^{i}$, on which $S U(2)$ acts with weight $i$.

The following linear-algebraic lemma allows one to compute $\Lambda_{+}^{i}$ explicitly

LEMMA 5.1. In the above assumptions, let $I$ be an induced complex structure, and $\mathbb{H}_{I}$ the quaternion space, considered as a 2-dimensional complex vector space with the complex structure induced by $I$. Denote by $\Lambda_{I}^{p, 0}(M)$ the space of $(p, 0)$-forms on $(M, I)$. The space $\mathbb{H}_{I}$ is equipped with a natural action of $S U(2)$. Consider $\Lambda_{I}^{p, 0}(M)$ as a representation of $S U(2)$, with trivial group action. Then, there is a canonical isomorphism

$$
\Lambda_{+}^{p}(M) \cong S_{\mathbb{C}}^{p} \mathbb{H}_{I} \otimes_{\mathbb{C}} \Lambda_{I}^{p, 0}(M)
$$

where $S_{\mathbb{C}}^{p} \mathbb{H}_{I}$ denotes a $p$-th symmetric power of $\mathbb{H}_{I}$. Moreover, the $S U(2)$-action on $\Lambda_{+}^{p}(M)$ is compatible with the isomorphism (5.1).

Proof. This is [V3], Lemma 8.1.

Consider an $S U(2)$-invariant decomposition

$$
\Lambda^{p}(M)=\Lambda_{+}^{p}(M) \oplus V^{p},
$$

where $V^{p}$ is the sum of all $S U(2)$-subspaces of $\Lambda^{p}(M)$ of weight less than $p$. Using the decomposition (5.2), we define the quaternionic Dolbeault differential $d_{+}$: $\Lambda_{+}^{*}(M) \longrightarrow \Lambda_{+}^{*}(M)$ as a composition of de Rham differential and projection of to $\Lambda_{+}^{*}(M) \subset \Lambda^{*}(M)$. Since the de Rham differential cannot increase the $S U(2)$-weight of a form more than by $1, d$ preserves the subspace $V^{*} \subset \Lambda^{*}(M)$. Therefore, $d_{+}$is a differential in $\Lambda_{+}^{*}(M)$.

DEFINITION 5.2. Let

$$
\Lambda^{0} M \stackrel{d_{+}}{\longrightarrow} \Lambda^{1} M \stackrel{d_{+}}{\longrightarrow} \Lambda_{+}^{2} M \stackrel{d_{+}}{\longrightarrow} \Lambda_{+}^{3} M \stackrel{d_{+}}{\longrightarrow} \ldots
$$

be the differential graded algebra constructed above ${ }^{1}$. It is called the quaternionic Dolbeault complex, or Salamon complex.

5.2. Hodge decomposition for the quaternionic Dolbeault complex. Let $M$ be a hypercomplex manifold, and $I$ an induced complex structure. Consider the operator $a d I: \Lambda^{*}(M) \longrightarrow \Lambda^{*}(M)$ mapping a $(p, q)$-form $\eta$ to $\sqrt{-1}(p-q) \eta$. By definition, adI belongs to the Lie algebra $\mathfrak{s u}(2)$ acting on $\Lambda^{*}(M)$ in the standard

\footnotetext{
${ }^{1}$ We identify $\Lambda^{0} M$ and $\Lambda_{+}^{0} M, \Lambda^{1} M$ and $\Lambda_{+}^{1} M$.
} 
way. Therefore, adI preserves the subspace $\Lambda_{+}^{*}(M) \subset \Lambda^{*}(M)$. We obtain the Hodge decomposition

$$
\Lambda_{+}^{*}(M)=\oplus_{p, q} \Lambda_{+, I}^{p, q}(M) .
$$

DEFINITION 5.3. The decomposition

$$
\Lambda_{+}^{*}(M)=\oplus_{p, q} \Lambda_{+, I}^{p, q}(M)
$$

is called the Hodge decomposition for the quaternionic Dolbeault complex.

The following claim is trivial

Claim 5.4. Given a hypercomplex manifold $M$ and an induced complex structure $I$, the following subspaces of $\Lambda^{*}(M)$ coinside:

$$
\Lambda_{+, I}^{p, 0}(M)=\Lambda_{I}^{p, 0}(M),
$$

where $\Lambda_{I}^{p, 0}(M)$ denotes the space of all $(p, 0)$-forms.

Proof. Immediately follows from Lemma 5.1.

5.3. Quaternionic Dolbeault bicomplex: explicit description. Let $M$ be a hypercomplex manifold, $I$ an induced comlex structure, and $I, J, K \in \mathbb{H}$ the standard triple of induced complex structures. Clearly, $J$ acts on the complexified co tangent space $\Lambda^{1} M \otimes \mathbb{C}$ mapping $\Lambda_{I}^{0,1}(M)$ to $\Lambda_{I}^{1,0}(M)$. Consider a differential operator

$$
\partial_{J}: C^{\infty}(M) \longrightarrow \Lambda_{I}^{1,0}(M)
$$

mapping $f$ to $J(\bar{\partial} f)$, where $\bar{\partial}: C^{\infty}(M) \longrightarrow \Lambda_{I}^{0,1}(M)$ is the standard Dolbeault differential on a Kähler manifold $(M, I)$. We extend $\partial_{J}$ to a differential

$$
\partial_{J}: \Lambda_{I}^{p, 0}(M) \longrightarrow \Lambda_{I}^{p+1,0}(M)
$$

using the Leibniz rule.

Proposition 5.5. Let $M$ be a hypercomplex manifold, $I$ an induced complex structure, $I, J, K$ the standard basis in quaternion algebra, and

$$
\Lambda_{+}^{*}(M)=\oplus_{p, q} \Lambda_{I,+}^{p, q}(M)
$$

the Hodge decomposition of the quaternionic Dolbeault complex. Then there exists a canonical isomorphism

$$
\Lambda_{I,+}^{p, q}(M) \cong \Lambda_{I}^{p+q, 0}(M) .
$$

Under this identification, the quaternionic Dolbeault differential

$$
d_{+}: \Lambda_{I,+}^{p, q}(M) \longrightarrow \Lambda_{I,+}^{p+1, q}(M) \oplus \Lambda_{I,+}^{p, q+1}(M)
$$

corresponds to a sum

$$
\partial \oplus \partial_{J}: \Lambda_{I}^{p+q, 0}(M) \longrightarrow \Lambda_{I}^{p+q+1,0}(M) \oplus \Lambda_{I}^{p+q+1,0}(M) .
$$


Proof. This is Proposition 8.13 of [V3].

The statement of Proposition 5.5 can be represented by the following diagram
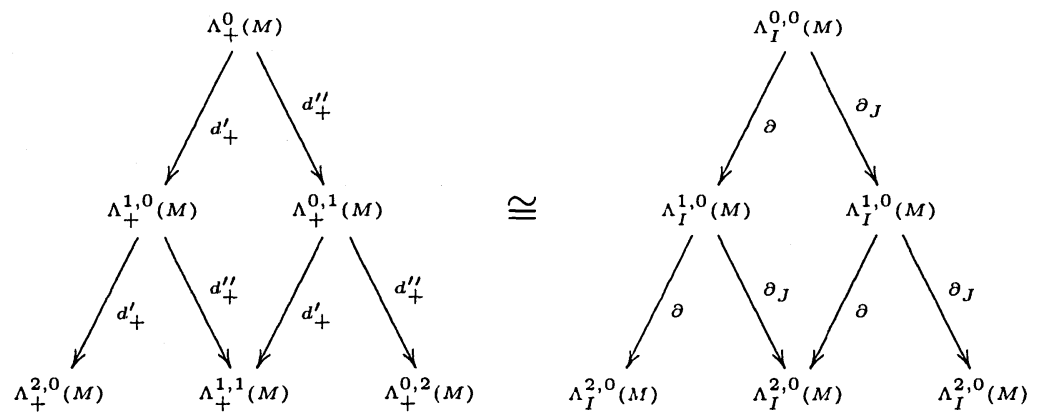

where $d_{+}=d_{+}^{\prime}+d_{+}^{\prime \prime}$ is the Hodge decomposition of the quaternionic Dolbeault differential.

REMARK 5.6. Since $d_{+}^{2}=0$, we obtain that $\partial$ and $\partial_{J}$ anti-commute:

$$
\left\{\partial, \partial_{J}\right\}=0
$$

Further on in this paper, only (5.5) will be used; however, the analogy between the quaternionic Dolbeault complex and the de Rham algebra of a Kähler manifold is implicit in our construction.

The definition of an HKT-manifold can be reformulated in terms of a quaternionic Dolbeault complex as follows.

THEOREM 5.7. Let $M$ be a hypercomplex Hermitian manifold, $I$ an induced complex structure, $\omega_{I}$ the corresponding 2 -form, and $d_{+}$the quaternionic Dolbeault differential. Then $\partial \Omega=0$ if and only if $d_{+} \omega_{I}=0$.

Proof. Let $I, J, K$ be the standard basis in quaternions. Consider $\omega_{I}$ as an element in $\Lambda_{I,+}^{1,1}(M)$. Using Proposition 5.5, we identify $\Lambda_{I,+}^{1,1}(M)$ and $\Lambda_{I,+}^{2,0}(M)$. Under this identification, $\omega_{I}$ corresponds to the standard $(2,0)$-form $\Omega$ (see [V3]). Therefore, $\omega_{I}$ is $d_{+}$-closed if and only if $\Omega$ is $d_{+}$-closed.

Clearly,

$$
\partial_{J} \Omega=J(\overline{\partial \Omega})
$$

(see, e.g. Proposition 7.1), hence $\partial \Omega=0$ if and only if $\partial_{J} \Omega=0$. By Proposition 5.5, we find that $\partial \Omega=0$ if and only if $d_{+} \Omega=0$. This proves Theorem 5.7.

REMARK 5.8. By Theorem 3.7, $M$ is an HKT-manifold if and only if $\partial \Omega=0$. This allows one to interpret Theorem 5.7 in terms of the HKT-condition: a manifold $M$ is HKT if and only if $d_{+} \omega_{I}=0$. This statement is analogous to the definition of a Kähler manifold in terms of a symplectic form: "a complex Hermitian manifold is Kähler if and only if the standard $(1,1)$-form $\omega$ is closed". 
REMARK 5.9. One can easily deduce Theorem 3.7 (the HKT conditions) from Theorem 5.7. Consider a hypercomplex Hermitian manifold which satisfies $\partial \Omega=0$. Then $d_{+} \Omega=0$ and $d_{+} \omega_{I}=0$. Therefore, for $\omega=\omega_{I}, \omega_{J}, \omega_{K}$, the differential $d \omega$ is of weight 1 with respect to the $S U(2)$-action. This is the condition (ii) of Theorem 3.7. In other words, Theorem 5.7 allows us to check that Theorem 3.7 (i) $\Leftrightarrow$ Theorem 3.7 (ii).

The implication Theorem 3.7 (ii) $\Rightarrow$ Theorem 3.7 (iii) is standard. The torsion forms of the connections $\nabla_{I}, \nabla_{J}, \nabla_{K}$ are equal to $I d \omega_{I}, J d \omega_{J}, K d \omega_{K}$. Suppose that

$$
I d \omega_{I}=J d \omega_{J}=K d \omega_{K}
$$

that is, the torsion forms of $\nabla_{I}, \nabla_{J}, \nabla_{K}$ are equal. Substracting the torsion form from $\nabla_{I}, \nabla_{J}, \nabla_{K}$, we obtain three torsion-free orthogonal connections on $M$. These connections are equal to Levi-Civita connection, because Levi-Civita connection is the unique orthogonal torsion-free connection. This implies $\nabla_{I}=\nabla_{J}=\nabla_{K}$. Therefore, a hypercomplex Hermitian manifold is HKT if and only if (5.6) holds. When $I d \omega_{I}$, $J d \omega_{J}, K d \omega_{K}$ all have weight one with respect to $S U(2),(5.6)$ follows from elementary calculation; converse is also clear.

\section{The hyperkähler-de Rham superalgebra.}

6.1. The so(1,4)-action on $\Lambda^{*}(M)$. Let $M$ be a hyperkähler manifold, $I, J, K$ induced complex structures giving a standard basis in quaternions, and $\omega_{I}, \omega_{J}, \omega_{K}$ the corresponding 2-forms (Definition 2.6). Denote by $L_{I}, L_{J}, L_{K}: \Lambda^{*}(M) \longrightarrow \Lambda^{*+2}(M)$ the operators of exterior multiplication by $\omega_{I}, \omega_{J}, \omega_{K}$. Let

$$
\Lambda_{I}:=* L_{I} *, \Lambda_{J}:=* L_{J} *, \Lambda_{K}:=* L_{K} *
$$

be the Hermitian adjoint operators, and $H$ the standard Hodge operator; we have three $\mathfrak{s l}(2)$-triples $\left\langle L_{I}, \Lambda_{I}, H\right\rangle,\left\langle L_{J}, \Lambda_{J}, H\right\rangle,\left\langle L_{K}, \Lambda_{K}, H\right\rangle$ acting on $\Lambda^{*}(M)$.

Given an induced complex structure $L \in \mathbb{H}$, consider an operator

$$
a d L: \Lambda_{L}^{p, q}(M) \longrightarrow \Lambda_{L}^{p, q}(M), \quad \eta \longrightarrow \sqrt{-1}(p-q) \eta .
$$

Clearly, $a d L$ is the Lie algebra element corresponding to the $U(1)$-action which induces the Hodge decomposition. Therefore, the endomorphism adL belongs to the Lie algebra of the standard $S U(2)$-action on $H^{*}(M)$; moreover, this Lie algebra is generated by $a d L$, for $L=I, J, K$.

The following theorem is implied by an easy linear-algebrain computation.

THEOREM 6.1. Consider the 10-dimensional vector space space $\mathfrak{a} \subset \operatorname{End}\left(\Lambda^{*}(M)\right)$ generated by $H, L_{X}, \Lambda_{X}, a d X$, for $X=I, J, K$. Then $\mathfrak{a}$ is a Lie subalgebra of $\operatorname{End}\left(\Lambda^{*}(M)\right)$. Moreover, $\mathfrak{a}$ is isomorphic to $\mathfrak{s o}(1,4)$.

Proof. See [V0]. 
6.2. Hyperkähler-de Rham superalgebra: the definition. Consider the 2-dimensional quaternionic vector space $W$ equipped with a quaternionic Hermitian metrics of signature,+- . Denote the group of quaternionic-linear isometries of $W$ by $S U(1,1, \mathbb{H})$. The group $S U(1,1, \mathbb{H})$ acts on $W$ in a tautological way. Using the classical isomorphism

$$
S U(1,1, \mathbb{H}) \cong \operatorname{Spin}(1,4),
$$

we may consider $W$ as a representation of $\mathfrak{a}=\mathfrak{s o}(1,4)$.

Assume that the grading of $W$ is odd. Let $\mathcal{H}:=W \oplus \mathbb{R} D$ be a direct sum of $W$ and an even 1-dimensional vector space. Consider the map

$$
(\cdot, \cdot) D: W \times W \longrightarrow \mathbb{R} D
$$

putting $v, v^{\prime} \in W$ to $\left(v, v^{\prime}\right)_{D} \subset \mathcal{H}$, where $(\cdot, \cdot)$ is the Hermitian form on $W$, and $D$ the generator of $\mathbb{R} D \subset \mathcal{H}$.

We introduce a structure of a Lie superalgebra on $\mathcal{H}$ as follows: for odd elements $v, v^{\prime} \in W$, we have

$$
\left\{v, v^{\prime}\right\}=\left(v, v^{\prime}\right)_{D}
$$

and a supercommutator of $D$ with anything is zero. Such an algebra is called the odd Heisenberg algebra; it is a graded version of a usual Heisenberg algebra.

Clearly, $\mathfrak{a}=\mathfrak{s o}(1,4)$ acts on $\mathcal{H}$ by automorphisms.

Definition 6.2. Let $\mathfrak{a} \rtimes \mathcal{H}$ be a semidirect product of $\mathfrak{a}$ and $\mathcal{H}$. Then $\mathfrak{a} \rtimes \mathcal{H}$ a hyperkähler-de Rham superalgebra.

THEOREM 6.3. ([FKS]) Let $M$ be a hyperkähler manifold, $\mathfrak{a} \cong \mathfrak{s o}(1,4)$ the Lie algebra constructed in Theorem 6.1 and acting on the differential forms, $\mathfrak{a} \subset$ $\operatorname{End}\left(\Lambda^{*}(M)\right)$. Consider the Lie superalgebra $\mathfrak{g} \subset \operatorname{End}\left(\Lambda^{*}(M)\right)$, generated by the even subspace $\mathfrak{a} \subset \operatorname{End}\left(\Lambda^{*}(M)\right)$ and the odd vector $d \in \operatorname{End}\left(\Lambda^{*}(M)\right)$, where $d: \Lambda^{*}(M) \longrightarrow \Lambda^{*+1}(M)$ is the de Rham differential. Then $\mathfrak{g}$ is naturally isomorphic to the hyperkähler-de Rham superalgebra (Definition 6.2).

Proof. Let $I_{1} \in \mathbb{H}$ be an induced complex structure. Consider the differential $d_{I_{1}}:=-I_{1} \circ d \circ I_{1} \cdot{ }^{1}$. Since $d_{I_{1}}=\sqrt{-1}\left[a d I_{1}, d\right]$, the differential $d_{I_{1}}$ belongs to $\mathfrak{g}$. Let $d_{I_{1}}^{*}:=-* d_{I_{1}} *$. By Kodaira identities (see (4.2)), we have

$$
d_{I_{1}}^{*}=-\left[\Lambda_{I_{1}}, d\right] \text {. }
$$

Therefore, the vectors $d_{I_{1}}^{*}$ belong to $\mathfrak{g}$.

Let $W \subset \mathfrak{g}$ be the 8-dimensional odd subspace spanned by

$$
d, d_{I}, d_{J}, d_{K}, d^{*}, d_{I}^{*}, d_{J}^{*}, d_{K}^{*}
$$

Using the Kodaira relations, it is easy to check that all pairwise commutators of the vectors $\left\langle d, d_{I}, d_{J}, d_{K}, d^{*}, d_{I}^{*}, d_{J}^{*}, d_{K}^{*}\right\rangle$ vanish, except $\left\{d, d^{*}\right\},\left\{d_{I}, d_{I}^{*}\right\},\left\{d_{J}, d_{J}^{*}\right\}$, $\left\{d_{K}, d_{K}^{*}\right\}$. Moreover, we have

$$
\left\{d, d^{*}\right\}=\left\{d_{I}, d_{I}^{*}\right\}=\left\{d_{J}, d_{J}^{*}\right\}=\left\{d_{K}, d_{K}^{*}\right\}=\Delta,
$$

\footnotetext{
${ }^{1}$ In the Kähler situation, this differential was denoted by $d^{c}$. Over a hyperkähler manifold, one needs to emphasize the dependence from the choice of the induced complex structure $I_{1}$.
} 
where $\Delta=d d^{*}+d^{*} d$ is the usual Laplace operator on the differential forms.

By the Kodaira relations, the Laplacian $\Delta$ commutes with $d, d_{I_{1}}, L_{I_{1}}$ and $\Lambda_{I_{1}}$, for any induced Kähler structure $I_{1}$. Therefore, $\Delta$ belongs to the centre of $\mathfrak{g}$.

For any two vectors $v, v^{\prime} \in W$, the super-commutator $\left\{v, v^{\prime}\right\}$ is proportional to the Laplacian. This allows one to speak of the quotient $\frac{\left\{v, v^{\prime}\right\}}{\Delta} \in \mathbb{R}$. Consider the pairing

$$
(\cdot, \cdot): W \times W \longrightarrow \mathbb{R}
$$

mapping $v, v^{\prime}$ to $\frac{\left\{v, v^{\prime}\right\}}{\Delta}$. Since $\mathfrak{a} \cong \mathfrak{s o}(1,4)$ preserves the Laplacian, a acts on $W$ preserving the pairing $(\cdot, \cdot)$. An elementary calculation is used to check that $(\cdot, \cdot)$ has signature $(+,+,+,+,-,-,-,-)$. The Lie algebra $\mathfrak{a}=\mathfrak{s u}(1,1, \mathbb{H})$ acts on $W$ as on its fundamental representation; this identifies $W$ and $\mathbb{H} \oplus \mathbb{H}$. Since $\Delta$ lies in the center of $\mathfrak{g}$, the subalgebra $\mathcal{H}:=W \oplus \mathbb{R} \Delta \subset \mathfrak{g}$ is isomorphic to the odd Heisenberg algebra; this sublalgebra is preserved by $\mathfrak{a}=\mathfrak{s o}(1,4) \subset \mathfrak{g}$, and $\mathfrak{g}$ is generated by $\mathfrak{a}$ and $\mathcal{H}$. Therefore, $\mathcal{H}$ is an ideal of $\mathfrak{g}$, and $\mathfrak{g}$ is a semidirect product of $\mathcal{H}$ and $\mathfrak{a}$.

We have proven Theorem 6.3.

6.3. Dolbeault complex and the hyperkähler-de Rham superalgebra. Let $M$ be a hyperkähler manifold, $I, J, K$ the standard triple of induced complex structures, $I \circ J=-J \circ I=K$, and $\mathfrak{g}=\mathfrak{a} \rtimes \mathcal{H}$ the Kähler-de Rham superalgebra acting on $\Lambda^{*}(M)$.

Let $\Omega=\frac{1}{2}\left(\omega_{J}+\sqrt{-1} \omega_{K}\right)$ be the standard $(2,0)$-form on $(M, I)$, and $L_{\Omega}$ the operator of multiplication by $\Omega$. Consider the Hermitian adjoint operator $\Lambda_{\Omega}=$ $* L_{\Omega} *$, and let $H_{\Omega}$ be their commutator. Using Theorem 6.1 , it is easy to check that $\left\langle L_{\Omega}, \Lambda_{\Omega}, H_{\Omega}\right\rangle$ is an $\mathfrak{s l}(2)$-triple (see [V0]). Moreover, $H_{\Omega}$ maps $\eta \in \Lambda_{I}^{p, q}(M)$ to $(n-p) \eta$, where $n=\operatorname{dim}_{\mathbb{H}} M$.

The main result of this Subsection is the following proposition.

Proposition 6.4. Let $M$ be a hyperkähler manifold, $I, J, K$ the standard triple of induced complex structures, $I \circ J=-J \circ I=K$, and $\mathfrak{g}=\mathfrak{a} \rtimes \mathcal{H}$ the hyperkähler-de Rham superalgebra acting on $\Lambda^{*}(M)$. Consider an 8-dimensional subspace $\mathfrak{g}_{I}^{*, 0} \subset \mathfrak{g}$ spanned by the vectors

$$
L_{\Omega}, \Lambda_{\Omega}, H_{\Omega}, \partial, \partial^{*}, \partial_{J}, \partial_{J}^{*}, \Delta .
$$

Then $\mathfrak{g}_{I}^{*, 0}$ is closed under the super-commutator. Moreover, $\mathfrak{g}_{I}^{*, 0}$ is naturally isomorphic to the Kähler-de Rham superalgebra.

REMARK 6.5. In fact, the Dolbeault complex of a hyperkähler manifold is on most counts similar to the de Rham complex of a Kähler manifold. This analogy was a driving engine behind [V1].

Proof of Proposition 6.4. Let $W$ be the odd part of the hyperkähler-de Rham superalgebra $\mathfrak{g}$ :

$$
W:=\left\langle d, d_{I}, d_{J}, d_{K}, d^{*}, d_{I}^{*}, d_{J}^{*}, d_{K}\right\rangle
$$

Then, $W$ admits a Hodge decomposition

$$
W=W^{1,0} \oplus W^{0,1} \oplus W^{-1,0} \oplus W^{0,-1}
$$


with each component 2-dimensional, and generated by $\partial, \partial_{J}$, and their complex adjoint and Hermitian conjugate as follows:

$$
\begin{aligned}
& W^{1,0}=\left\langle\partial, \partial_{J}\right\rangle, \quad W^{0,1}=\left\langle\bar{\partial}, \bar{\partial}_{J}\right\rangle, \\
& W^{-1,0}=\left\langle\partial^{*}, \partial_{J}^{*}\right\rangle, \quad W^{0,-1}=\left\langle\bar{\partial}^{*}, \bar{\partial}_{J}^{*}\right\rangle .
\end{aligned}
$$

Clearly, the odd part of $\mathfrak{g}_{I}^{*, 0}$ coinsides with $W^{1,0} \oplus W^{-1,0}$. Since the $\mathfrak{s l}(2)$-triple $\left\langle L_{\Omega}, \Lambda_{\Omega}, H_{\Omega}\right\rangle$ maps $(p, q)$-forms to $\left(p^{\prime}, q\right)$-forms, it preserves $W^{1,0} \oplus W^{-1,0}$. The pairwise super-commutators of odd elements in $\mathfrak{g}$ are proportional to the Laplacian $\Delta$. Therefore, the space $\mathfrak{g}_{I}^{*, 0}$ is closed under super-commutator.

To prove that $\mathfrak{g}_{I}^{* 0}$ is isomorphic to the Kähler-de Rham superalgebra, we use Theorem 4.3. It suffices to show that the vectors $\partial, \partial_{J}$ anticommute ${ }^{2}$ with themselves and with each other, and commute with $L_{\Omega}$.

These vectors anticommute by Kodaira relations (see Theorem 6.3). Since $\Omega$ is closed under the differentials $d, d_{I}, d_{J}, d_{K}$, we have

$$
\left[L_{\Omega}, \partial\right]=\left[L_{\Omega}, \partial_{J}\right]=0 .
$$

Applying Theorem 4.3 , we find that $\mathfrak{g}_{I}^{*, 0}$ is isomorphic to the Kähler-de Rham superalgebra. Proposition 6.4 is proven.

In HKT-geometry, $d \Omega=0$ and the hyperkähler-de Rham superalgebra does not act on $\Lambda^{*}(M)$. However, due to the HKT-relation $\partial \Omega=0$, there exists the Kähler-de Rham superalgebra action on $\Lambda^{*, 0}(M, I)$. This structure is the main object of this paper.

7. Lie superalgebra of an HKT-manifold. Let $M$ be a hypercomplex Hermitian manifold, $I$ an induced complex structure, and $\omega_{I}$ the corresponding nondegenerate 2 -form (Definition 2.6). Denote by $L_{I}: \Lambda^{*}(M) \longrightarrow \Lambda^{*+2}(M)$ the operator of multiplication by $\omega_{I}$, and let $\Lambda_{I}$ the Hermitian adjoint operator (Subsection 6.3). Clearly, the $\mathfrak{s o}(1,4)$-action (Theorem 6.1 ) is valid for hypercomplex Hermitian manifolds as well. However, there is no reason for Kodaira relations to hold; this is why there is no Hodge decomposition and no $\mathfrak{s o}(1,4)$-action on the cohomology of a hyperkähler manifold.

However, the HKT relation $\partial \Omega=0$ (Theorem 3.7 (i)) can be directly translated to a statement about the Lie superalgebra.

Fix the standard quaternion basis $I, J, K$,

$$
I^{2}=J^{2}=K^{2}=-1, I J=-J I=K,
$$

Let $\Omega:=\frac{1}{2}\left(\omega_{J}+\sqrt{-1} \omega_{K}\right)$ be the corresponding $(2,0)$-form (Definition 2.6),

$$
L_{\Omega}: \Lambda^{*}(M) \longrightarrow \Lambda^{*+2}(M)
$$

the operator of multiplication by $\Omega$, and

$$
\Lambda_{\Omega}: \Lambda^{*}(M) \longrightarrow \Lambda^{*-2}(M)
$$

the Hermitian adjoint operator: $\Lambda_{\Omega}=* L_{\Omega} *$.

\footnotetext{
${ }^{2}$ That is, commute in the sense of superalgebra.
} 
From Theorem 6.1 it follows that the commutator $H_{\Omega}:=\left[L_{\Omega}, \Lambda_{\Omega}\right]$ is a scalar operator, mapping a $(p, q)$-form $\eta$ to $(n-p) \eta$, where $n=\operatorname{dim}_{\mathbb{H}} M$. Therefore, $L_{\Omega}$, $\Lambda_{\Omega}, H_{\Omega}$ form an $\mathfrak{s l}(2)$-triple on the standard Lie algebra $\mathfrak{s o}(1,4)$ acting on $\Lambda^{*}(M)$.

Consider the operator $J$ acting on differential forms as

$$
J\left(d x_{1} \wedge d x_{2} \wedge \ldots \wedge d x_{n}\right)=J\left(d x_{1}\right) \wedge J\left(d x_{2}\right) \wedge \ldots \wedge J\left(d x_{n}\right)
$$

where $d x_{1} \in \Lambda^{1}(M)$, and the action of $J$ on $\Lambda^{1}(M)$ is determined from the quaternion structure. Since $J$ and $I$ anticommute, $J$ maps $(p, q)$-forms to $(q, p)$-forms.

Let $\partial: \Lambda^{p, q}(M) \longrightarrow \Lambda^{p+1, q}(M)$ be the Dolbeault differential, and

$$
\partial_{J}: \Lambda^{p, q}(M) \longrightarrow \Lambda^{p+1, q}(M)
$$

the differential obtained as

$$
\partial_{J}:=-J \circ \bar{\partial} \circ J
$$

Proposition 7.1. Let $M$ be a hypercomplex Hermitian manifold, with $L_{\Omega}, \partial$ and $\partial_{J}$ defined as above. Then the HKT-relation $\partial \Omega=0$ (Theorem 3.7 (i)) is equivalent to the following Lie algebra relations

$$
\left[L_{\Omega}, \partial\right]=\left[L_{\Omega}, \partial_{J}\right]=0 .
$$

Proof. Clearly, $\partial \Omega=0$ is equivalent to $\left[L_{\Omega}, \partial\right]=0$. However, $J(\Omega)=\bar{\Omega}$, hence

$$
\partial_{J} \Omega=J(\overline{\partial \Omega})=J(\overline{\partial \Omega}) .
$$

Therefore, $\partial_{J} \Omega=0$ if and only if $\partial \Omega=0$. We obtain that $\partial \Omega=0$ implies $\left[L_{\Omega}, \partial_{J}\right]=0$. This proves Proposition 7.1.

Corollary 7.2. Let $M$ be an HKT-manifold, with $L_{\Omega}, \Lambda_{\Omega}, H_{\Omega}, \partial$ and $\partial_{J}$ defined as above. Consider the Lie superalgebra $\mathfrak{g}$ generated by these operators. Then $\mathfrak{g}$ is isomorphic to the Kähler-de Rham superalgebra.

Proof. By Proposition 7.1, we have

$$
\left[L_{\Omega}, \partial\right]=\left[L_{\Omega}, \partial_{J}\right]=0
$$

By (5.5), $\partial$ and $\partial_{J}$ (anti-)commute:

$$
\left\{\partial, \partial_{J}\right\}=0
$$

In Subsection 6.3, we have seen that $H_{\Omega}$ acts on $(p, q)$-forms as a muptiplication by $p-2 n$. Therefore,

$$
\left[H_{\Omega}, \partial\right]=\partial, \quad\left[H_{\Omega}, \partial_{J}\right]=\partial_{J} .
$$

From these relations and Theorem 4.3 we obtain that $\mathfrak{g}$ is isomorphic to the Kähler-de Rham superalgebra.

8. Kodaira relations in a differential graded algebra. 
8.1. Differential operators in graded commutative rings. Let $A$ be a graded commutative algebra over $\mathbb{C}$. We define the space $D_{i}(A) \subset \operatorname{Hom}_{\mathbb{C}}(A, A)$ of differential operators of order $\leqslant i$ recursively as follows.

Definition 8.1. (A. Grothendieck)

(i) The space $D_{0}(A)$ of zero-order differential operators is canonically identified with $A$ acting on itself by multiplication.

(ii) $D_{i}(A)$ is the space of all $\mathbb{C}$-linear maps $D: A \rightarrow A$ such that the supercommutator $[a, D]$ belongs to $D_{i-1}(A)$, for all $a \in D_{0}(A)$.

The differential operators form a Lie superalgebra.

We have

$$
D_{0}(A) \subset D_{1}(A) \subset D_{2}(A) \subset \ldots
$$

The following lemma is well known

LEMMA 8.2. We have

$$
\left[D_{i}(A), D_{j}(A)\right] \subset D_{i+j-1}(A)
$$

As usually, we denote the parity of an element $v$ of a graded vector space by $\widetilde{v} \in\{0,1\} ; \widetilde{v}=0$ if $v$ is even, and $\widetilde{v}=1$ if $v$ is odd.

The notion of differential operators can be extended to $A$-modules in a usual fashion.

Definition 8.3. Let $M, N$ be $A$-mudules. We define the space

$$
D_{i}(M, N) \subset \operatorname{Hom}_{\mathbb{C}}(M, N)
$$

of differential operators of order $\leqslant i$ recursively as follows.

(i) The space $D_{0}(M, N)$ of zero-order differential operators is $\operatorname{Hom}_{A}(M, N)$

(ii) $D_{i}(M, N)$ is the space of all $\mathbb{C}$-linear maps $D: M \longrightarrow N$ such that for all $a \in A$, the supercommutator

$$
[a, D](m):=a \cdot D(m)+(-1)^{\tilde{a} \tilde{D}} D(a \cdot m)
$$

belongs to $D_{i-1}(M, N)$, for all $a \in A$.

REMark 8.4. Let $X$ be a smooth manifold, $A:=\Lambda^{*}(X), A_{0}:=C^{\infty}(X)$, and $M, N$ vector bundles on $X$, equipped with a structure of $A$-module. Denote $M, N$, considered as $C^{\infty}(X)$-modules, by $M_{C^{\infty}}, N_{C^{\infty}}$. Clearly, the $A_{0}$-differential operators $D_{i}\left(M_{C \infty}, N_{C^{\infty}}\right)$ are identified with the usual differential operators on the vector bundles corresponding to $M, N$. Since $A$-differential operator is necessarily an $A_{0^{-}}$ differential operator, the space $D_{i}(M, N)$ is a subspace of the space of $D_{i}\left(M_{C^{\infty}}, N_{C^{\infty}}\right)$ of differential operators on vector bundles. Generally speaking, the embedding

$$
D_{i}(M, N) \hookrightarrow D_{i}\left(M_{C \infty}, N_{C \infty}\right)
$$

is proper. 
The main example we are working with is the following.

Proposition 8.5. Let $X$ be a Riemannian manifold, $A=\Lambda^{*}(X)$ the algebra of differential forms on $X, *$ the Hodge star operator, and

$$
d^{*}:=-* d *: A \longrightarrow A
$$

the operator obtained as a Hermitian conjugate of the de Rham differential $d$. Then $d^{*}$ is a second order differential operator on the graded commutative algebra $A: d^{*} \in$ $D_{2}(A)$.

Proof. Consider the tensor product $A \otimes_{\mathbb{C}^{\infty} X} \Lambda^{1}(X)$ as an $A$-module. Let

$$
\nabla: A \longrightarrow A \otimes \mathbb{C}^{\infty} X \Lambda^{1}(X)
$$

be the Levi-Civita connection operator. The Leibniz formula implies

$$
\nabla \in D_{1}\left(A, A \otimes_{\mathbb{C}^{\infty} X} \Lambda^{1}(X)\right) .
$$

Since $\nabla$ is torsion-free, we have $d^{*}=\nabla \circ \iota$, where

$$
\iota: A \otimes_{\mathbb{C}^{\infty} X} \Lambda^{1}(X) \longrightarrow A
$$

is the operator of "inner multiplication":

$$
\iota(\eta \wedge v)=-*((* \eta) \wedge v)
$$

for all $\eta \in \Lambda^{*}(X), v \in \Lambda^{1}(X)$.

To prove Proposition 8.5 , it remains to show that the map

$$
\iota: A \otimes_{\mathbb{C}^{\infty} X} \Lambda^{1}(X) \longrightarrow A
$$

is a first-order differential operator of $A$-modules. This is clear, because

$$
\begin{aligned}
& \iota\left(d x_{1} \wedge d x_{2} \wedge \ldots \wedge d x_{k} \otimes v\right) \\
& \quad=\sum_{l=1}^{k}(-1)^{l-1} d x_{1} \wedge d x_{2} \wedge \ldots \wedge d x_{l-1} \wedge d x_{l+1} \ldots \wedge d x_{k} \cdot\left(v, d x_{l}\right)
\end{aligned}
$$

where $v$ and $d x_{i}$ are 1 -forms and $(\cdot, \cdot)$ is the Riemannian form.

CoROLlaRY 8.6. Let $\lambda$ be a differential form on $X, L_{\lambda}: A^{*} \longrightarrow A^{*}$ the operation of exterior multiplication by $\lambda$, and $D:=\left[L_{\lambda}, d^{*}\right] \in D_{*}(A)$ the super-commutator of $L_{\lambda}$ and $d^{*}$. Then $D \in D_{1}(A)$.

Proof. Follows from Lemma 8.2 and Proposition 8.5. $\square$ 
8.2. Kodaira relations and differential operators on hypercomplex Hermitian manifolds. Let $M$ be a hypercomplex Hermitian manifold, $I, J, K$ the standard triple of induced complex structures, and $\Omega \in \Lambda_{I}^{2,0}(M)$. the standard nondegenerate $(2,0)$-form on $(M, I)$. CVonsider be the Dolbeault differential

$$
\partial: \Lambda_{I}^{p, 0}(M) \longrightarrow \Lambda_{I}^{p+1,0}(M)
$$

on $(M, I)$, and let $\partial^{*}:=-* \partial *$ be its Hermitian adjoint. Consider the commutator

$$
\delta_{J}^{*}:=-\left[L_{\Omega}, \partial^{*}\right] .
$$

Proposition 8.7. In the above assumptions, the map

$$
\delta_{J}^{*}: \Lambda_{I}^{p, 0}(M) \longrightarrow \Lambda_{I}^{p+1,0}(M)
$$

is a first order differential operator (in the usual sense, that is, over $C^{\infty} M$ ) with the same symbol as the operator

$$
\partial_{J}: \Lambda_{I}^{p, 0}(M) \longrightarrow \Lambda_{I}^{p+1,0}(M)
$$

(Subsection 5.3). Moreover,

$$
\delta_{J}^{*} \in D_{1}\left(\Lambda_{I}^{*, 0}(M)\right),
$$

where $D_{1}\left(\Lambda^{*, 0}(M)\right)$ is the space of first order differential operators on the graded commutative algebra $\Lambda_{I}^{*, 0}(M)$ (Definition 8.1). ${ }^{1}$

Proof. A hypercomplex Hermitian manifolds admits a first order approximation by a flat hyperkähler manifold $M^{\prime}=\mathbb{H}^{n}$. On $M^{\prime}$, we have

$$
\left[L_{\Omega}, \partial^{*}\right]=-\partial_{J} .
$$

(Theorem 6.3). The symbol of $\delta_{J}^{*}$ does not change if we replace $M$ by its firstorder approximation. This proves the first statement of Proposition 8.7. The second statement of Proposition 8.7 is proven in exactly the same way as Corollary 8.6.

In assumptions of Proposition 8.7, consider the operator

$$
h:=\delta_{J}^{*}-\partial_{J} .
$$

By Proposition 8.7, $h$ is a $\mathbb{C}^{\infty} M$-linear operator on $\Lambda^{*}(M)$, which is a differential operator of first order over the commutative graded algebra $\Lambda_{I}^{*, 0}(M)$. By definition of first order differential operators, we have

$$
h\left(\eta_{1} \wedge \eta_{2}\right)=\quad(-1)^{\tilde{\eta}_{2}} \eta_{1} \wedge h\left(\eta_{2}\right)+h\left(\eta_{1}\right) \wedge \eta_{2}+(-1)^{\tilde{\eta}_{1}+\tilde{\eta}+2} \eta_{1} \wedge \eta_{2} \wedge h(1) .
$$

By (8.1), $h$ is determined by the values it takes on $\Lambda^{0}(M)$ and $\Lambda_{I}^{1,0}(M)$. This allows to compute $h$ explicitly, as follows.

\footnotetext{
${ }^{1}$ The differential operators over $C^{\infty} M$ and the differential operators on the graded commutative algebra $\Lambda_{I}^{*, 0}(M)$ are two distinct notions. See Remark 8.4 for a more explicit statement.
} 
Let $M, \operatorname{dim}_{\mathbb{H}} M=n$ be an HKT manifold, $I, J, K$ the standard quaternion triple, $\Omega \in \Lambda_{I}^{2,0}(M)$ the corresponding (2,0)-form, and

$$
L_{\Omega}: \Lambda_{I}^{*, 0}(M) \longrightarrow \Lambda_{I}^{*+2,0}(M)
$$

the operator of multiplication by $\Omega$. Let $\Omega^{n}$ be the corresponding nowhere degenerate section of the canonical bundle. Since $\bar{\partial} \Omega^{n}$ is a $(2 n, 1)$-form, and any $(2 n, 0)$-form on $(M, I)$ is proportional to $\Omega^{n}$, the form $\bar{\partial} \Omega^{n}$ is proportional to $\Omega^{n}$ :

$$
\bar{\partial} \Omega^{n}=\bar{\theta} \wedge \Omega^{n}
$$

(we denote the corresponding $(0,1)$-form by $\bar{\theta})$. Let

$$
J: \Lambda_{I}^{p, q}(M) \longrightarrow \Lambda_{I}^{q, p}(M)
$$

be the standard multiplicative map acting on $\Lambda^{1}(M)$ as $J$. Denote the form $J(\bar{\theta}) \in$ $\Lambda_{I}^{1,0}(M)$ by $\theta_{J}$.

THEOREM 8.8. Let $M$ be an HKT-manifold. In the above assumptions, consider the operator

$$
\delta_{J}^{*}:=-\left[L_{\Omega}, \partial^{*}\right]
$$

(Proposition 8.7). Let

$$
h:=\delta_{J}^{*}-\partial_{J}
$$

be the corresponding $C^{\infty}(M)$-linear operator. Then, for ald $\eta \in \Lambda_{I}^{*, 0}(M)$, we have

$$
h(\eta)=\theta_{J} \wedge \eta
$$

where $\theta_{J}$ is a 1 -form, determined by the hypercomplex Hermitian structure as above.

We prove Theorem 8.8 in Subsection 9.2.

\section{The Hodge *-operator on hypercomplex Hermitian manifolds.}

9.1. The Hodge $*$-operator explicitly. In this Subection, we perform explicit calculations related to the Hodge *-operator on a hypercomplex Hermitian manifold. These calculations are purely linear-algebraic; in fact, one can compute everything on a quaternionic Hermitian space.

Claim 9.1. Let $M$ be a hypercomplex Aermitian manifold, $I, J, K$ the standard quaternion triple, and $\Omega \in \Lambda_{I}^{2,0}(M)$ the corresponding $(2,0)$-form on $(M, I)$. Then (i) $* 1=\left(\frac{1}{n !}\right)^{2} \Omega^{n} \wedge \bar{\Omega}^{n}, \quad n=\operatorname{dim}_{\mathbb{H}} M$

(ii) $* \Omega=2 n\left(\frac{1}{n !}\right)^{2} \Omega^{n-1} \wedge \bar{\Omega}^{n}$

(iii) For any $(1,0)$-form $\eta$, we have

$$
* \eta=-\left(\frac{1}{n !}\right)^{2} \Omega^{n-1} \wedge \bar{\Omega}^{n} \wedge J(\bar{\eta}),
$$

where

$$
J: \Lambda_{I}^{p, q}(M) \longrightarrow \Lambda_{I}^{q, p}(M)
$$

is a standard multiplicative operator on differential forms acting on $\Lambda^{1}(M)$ as $J$. 
Proof. Claim 9.1 (i) is clear, because $* 1$ is by definition equal to the Riemannian volume form of $M$, and

$$
\operatorname{Vol}(M)=\frac{\Omega^{n} \bar{\Omega}^{n}}{n !^{2}} .
$$

To prove Claim 9.1 (ii), we write

$$
* \Omega=*\left(L_{\Omega} 1\right)=\Lambda_{\Omega}(* 1)=\Lambda_{\Omega} L_{\Omega}^{n} \bar{\Omega}^{n} \cdot \frac{1}{(n !)^{2}}
$$

(the last equation follows from Claim 9.1 (i)). Since $L_{\Omega}, \Lambda_{\Omega}, H_{\Omega}$ form an $\mathfrak{s l}(2)$-triple, and $\bar{\Omega}^{n}$ is a lowest vector of a weight $n$ representation, we have

$$
\Lambda_{\Omega} L_{\Omega}^{n} \bar{\Omega}^{n}=2 n \Omega^{n-1} \bar{\Omega}^{n}
$$

Comparing (9.2) and (9.1), we obtain Claim 9.1 (ii).

To prove Claim 9.1 (iii), we use the following elementary lemma.

LEMMA 9.2. Let $M$ be a hypercomplex Hermitian manifold, $I, J, K$ the standard quaternion triple, and $\Omega \in \Lambda_{I}^{2,0}(M)$ the corresponding $(2,0)$-form on $(M, I)$. Consider an arbitrary $(1,0)$-form $\eta \in \Lambda_{I}^{1,0}(M)$. Denote by

$$
L_{\eta}: \Lambda_{I}^{*, 0}(M) \longrightarrow \Lambda_{I}^{*+1,0}(M)
$$

the operator of exterior multiplication by $\eta$, and let $\Lambda_{\eta}:=-* L_{\eta} *$ be the Hermitian adjoint operator (so-called "inner multiplication by $\eta$ "). Let $L_{\Omega}$ be the operator of exterior multiplication by $\Omega$. Then

$$
\left[L_{\Omega}, \Lambda_{\eta}\right]=L_{J(\bar{\eta})}
$$

where

$$
J: \Lambda_{I}^{p, q}(M) \longrightarrow \Lambda_{I}^{q, p}(M)
$$

is the standard operator defined above.

Proof. Proven by a local computation.

Return to the proof of Claim 9.1 (iii). By Lemma 9.2 and Claim 9.1 (i), we have

$$
\begin{aligned}
*(\eta) & =* L_{\eta} 1=-\Lambda_{\eta}(* 1)=\frac{1}{(n !)^{2}} \Lambda_{\eta} L_{\Omega}^{n} \bar{\Omega}^{n} \\
& =-\frac{1}{(n !)^{2}} L_{J(\bar{\eta})} L_{\Omega}^{n-1} \bar{\Omega}^{n}=-\frac{1}{(n !)^{2}} J(\bar{\eta}) \wedge \Omega^{n-1} \wedge \Omega^{n} .
\end{aligned}
$$

This is exactly the statement of Claim 9.1 (iii). We proved Claim 9.1. 
9.2. The Hodge *-operator on HKT-manifolds and Dolbeault differential. We work in assumptions of Theorem 8.8. By Proposition 8.7 (see also (8.1)), to prove Theorem 8.8 , it suffices to check

$$
\delta_{J}^{*} \eta-\partial_{J} \eta=J(\bar{\theta}) \wedge \eta
$$

for $\eta \in \Lambda_{I}^{*, 0}(M)$ a 0 -form or an $(1,0)$-form. The operator $h=\delta_{J}^{*}-\partial_{J}$ is $C^{\infty}$-linear. Since $\partial_{J}^{2}=0$, the $\partial_{J}$-closed forms generate the space of all forms over $C^{\infty}(M)$. Therefore, to prove Theorem 8.8 , it suffices to check

$$
h(\eta)=J(\bar{\theta}) \wedge \eta
$$

for $\partial_{J}(\eta)=0, \eta \in \Lambda_{I}^{i, 0}(M), i=0,1$. For $\eta$ a $\partial_{J}$-closed function, we have

$$
\delta_{J}^{*} \eta=-\left[L_{\Omega}, \partial^{*}\right] \eta=\partial^{*}(\eta \Omega) .
$$

$\left(\partial^{*} \eta=0\right.$ because $\eta$ is 0 -form). Using Claim 9.1 (ii), we obtain

$$
\delta_{J}^{*} \eta=-2 n \frac{1}{(n !)^{2}} * \partial\left(\Omega^{n-1} \wedge \bar{\Omega}^{n}\right) .
$$

Using the definition of $\theta$ and the HKT equation $\partial \Omega=0$, we express the right hand side of (9.4) through $\theta$ as follows:

$$
\delta_{J}^{*} \eta=-2 n \frac{1}{(n !)^{2}} \eta *\left(\theta \wedge \Omega^{n-1} \wedge \bar{\Omega}^{n}\right) .
$$

We obtain

$$
h(\eta)=-\Lambda_{\theta}\left(*\left[2 n \frac{1}{(n !)^{2}} \Omega^{n-1} \wedge \bar{\Omega}^{n}\right]\right) \eta
$$

where $\eta$ is an arbitrary 0 -form. The expression in brackets is equal to $* \Omega$ as follows from Claim 9.1 (ii). Therefore,

$$
h(\eta)=-\Lambda_{\theta}(\Omega) \cdot \eta
$$

for any $\eta \in \Lambda^{0}(M)$. By Lemma 9.2 ,

$$
-\Lambda_{\theta}(\Omega)=\left[L_{\Omega}, \Lambda_{\theta}\right](1)=L_{J(\bar{\theta})}(1)=J(\bar{\theta}) .
$$

Comparing (9.5) and (9.6), we obtain

$$
h(\eta)=J(\bar{\theta}) \cdot \eta
$$

where $\eta$ is an arbitrary 0 -form. Now assume that $\eta \in \Lambda_{I}^{1,0}(M), \partial_{J}(\eta)=0$. By Claim 9.1 (iii), we have

$$
\begin{aligned}
\delta_{J}^{*} \eta & =-*\left[\Lambda_{\Omega}, \partial\right] * \eta \\
& =-\frac{1}{(n !)^{2}} *\left(\left[\Lambda_{\Omega}, \partial\right]\left(\Omega^{n-1} \wedge \bar{\Omega}^{n} \wedge J(\bar{\eta})\right)\right) .
\end{aligned}
$$

Since $\eta$ is $\partial_{J}$-closed, we have $\partial(J(\bar{\eta}))=0$. We have $\partial \Omega=0$ by HKT-equations. Since $J(\bar{\eta}) \wedge \bar{\Omega}^{n}$ is a lowest weight vector with respect to the $\mathfrak{s l}(2)$-action generated by $L_{\Omega}$, $\Lambda_{\Omega}, H_{\Omega}$, the form

$$
\Lambda_{\Omega}\left(\Omega^{n-1} \wedge \bar{\Omega}^{n} \wedge J(\bar{\eta})\right)
$$


is proportional to

$$
\Omega^{n-2} \wedge \bar{\Omega}^{n} \wedge J(\bar{\eta})
$$

Therefore, $\partial$ acts on $\Omega^{n-1} \wedge \bar{\Omega}^{n} \wedge J(\bar{\eta})$ and on $\Lambda_{\Omega}\left(\Omega^{n-1} \wedge \bar{\Omega}^{n} \wedge J(\bar{\eta})\right)$ as a multiplication by $\theta$, and we can replace $\partial$ in (9.7) by $L_{\theta}$. We obtain

$$
\delta_{J}^{*} \eta=\frac{1}{(n !)^{2}} *\left(\left[\Lambda_{\Omega}, L_{\theta}\right]\left(\Omega^{n-1} \wedge \bar{\Omega}^{n} \wedge J(\bar{\eta})\right)\right)=\left[L_{\Omega}, \Lambda_{\theta}\right] \eta
$$

On the other hand, $\left[L_{\Omega}, \Lambda_{\theta}\right]=L_{J(\bar{\theta})}$ (Lemma 9.2). We obtain

$$
\delta_{J}^{*} \eta=J(\bar{\theta}) \wedge \eta
$$

for any $\eta \in \Lambda_{I}^{1,0}(M), \partial_{J}(\eta)=0$. This finishes the proof of Theorem 8.8.

10. Hodge theory on $\Lambda^{p, 0}(M) \otimes K^{1 / 2}$.

10.1. The normalized Kähler-de Rham superalgebra of an HKT manifold. Let $M$ be an HKT manifold and

$$
\mathfrak{g}=\left\langle L_{\Omega}, \Lambda_{\Omega}, H_{\Omega}, \partial, \partial_{J}, \delta, \delta_{J}, \Delta\right\rangle
$$

be the corresponding Kähler-de Rham superalgebra (Corollary 7.2). Conjugating each generator of $\mathfrak{g}$ by $*$, we obtain another superalgebra

$$
\mathfrak{g}^{*}=\left\langle L_{\Omega}, \Lambda_{\Omega}, H_{\Omega}, \partial^{*}, \partial_{J}^{*}, \delta^{*}, \delta_{J}^{*}, \Delta^{*}\right\rangle
$$

which is naturally isomorphic to $\mathfrak{g}$. However, these algebras are distinct; to work with Hodge theory, we need to relate the Lie superalgebras and the Hodge *-operator.

In this section, we construct and study the "normalized" Kähler-de Rham superalgebra ${ }^{n} \mathfrak{g}$, which acts on $\Lambda_{I}^{*, 0}(M)$ in the same way as $\mathfrak{g}$ and $\mathfrak{g}^{*}$, and is fixed by *.

Consider the 4-dimensional vector space ${ }^{n} V$ spanned by the vectors

$$
\begin{aligned}
{ }^{n} \partial & :=\frac{\partial+\delta^{*}}{2},{ }^{n} \partial_{J}:=\frac{\partial_{J}+\delta_{J}^{*}}{2}, \\
{ }^{n} \partial^{*} & :=\frac{\partial^{*}+\delta^{*}}{2}, \quad{ }^{n} \partial_{J}^{*}:=\frac{\partial_{J}^{*}+\delta_{J}}{2} \in \operatorname{End}\left(\Lambda_{I}^{*, 0}(M)\right) .
\end{aligned}
$$

Consider the standard $\mathfrak{s l}(2)$-action on $\Lambda_{I}^{*, 0}(M)$ associated with the $\mathfrak{s l}(2)$-triple $L_{\Omega}, \Lambda_{\Omega}, H_{\Omega}$. Since this $\mathfrak{s l}(2)$-action exchanges $\partial$ with $\delta_{J}$ and $\delta^{*}$ with $\delta_{J}^{*}$, this action exchanges ${ }^{n} \partial$ with ${ }^{n} \partial_{J}^{*}$ :

$$
\left[L_{\Omega},{ }^{n} \partial_{J}^{*}\right]={ }^{n} \partial, \quad\left[\Lambda_{\Omega},{ }^{n} \partial_{J}^{*}\right]=-{ }^{n} \partial .
$$

In other words, the standard $\mathfrak{s l}(2)$-triple

$$
\left\langle L_{\Omega}, \Lambda_{\Omega}, H_{\Omega}\right\rangle
$$

preserves the 2-dimensional space $\left\langle{ }^{n} \partial,{ }^{n} \partial_{J}^{*}\right\rangle$ spanned by ${ }^{n} \partial,{ }^{n} \partial_{J}^{*}$. Similarly, it preserves the space $\left\langle{ }^{n} \partial^{*},{ }^{n} \partial_{J}\right\rangle$.

We obtain that this $\mathfrak{s l}(2)$-action preserves the space ${ }^{n} V \subset \operatorname{End}\left(\Lambda_{I}^{*, 0}(M)\right)$, spanned by these two 2-dimensional spaces defined above. 
Let $\theta \in \Lambda_{I}^{1,0}(M)$ be a 1 -form defined by the formula

$$
\partial \bar{K}=\theta \wedge \bar{K}
$$

where $\bar{K} \in \Lambda_{I}^{0,2 n}(M), \bar{K}=\bar{\Omega}^{n}, n=\operatorname{dim}_{\mathbb{H}} M$ the natural section of the line bundle $\Lambda_{I}^{0,2 n}(M)$ determined by the standard nowhere degenerate $(0,2)$-form $\bar{\Omega}$. Denote by $\theta_{J}$ the form $J(\bar{\theta}) \in \Lambda_{I}^{1,0}(M)$. By Theorem 8.8, we have

$$
{ }^{n} \partial-\delta^{*}=\theta, \quad{ }^{n} \partial_{J}-\delta_{J}^{*}=\theta_{J} .
$$

This implies

$$
{ }^{n} \partial=\partial+\frac{1}{2} \theta, \quad{ }^{n} \partial_{J}=\partial_{J}+\frac{1}{2} \theta_{J} .
$$

By definition of $\theta$, we have

$$
\begin{aligned}
0=\partial \partial \bar{K} & =\partial(\theta \wedge \bar{K}) \\
& =\partial(\theta) \wedge \bar{K}-\theta \wedge \partial \bar{K}=\partial(\theta) \wedge \bar{K}-\theta \wedge \theta \wedge \bar{K} \\
& =\partial(\theta) \wedge \bar{K} .
\end{aligned}
$$

Therefore, $\theta$ is $\partial$-closed, and we have

$$
{ }^{n} \partial^{2}=\left(\partial-\frac{1}{2} \theta\right)^{2}=0
$$

Twisting this equation with $J$, we obtain ${ }^{n} \partial_{J}^{2}=0$. Finally, $\partial$ and $\partial_{J}$ anticommute (5.5), and we have

$$
\begin{aligned}
0=\partial \partial_{J} \bar{K}+\partial_{J} \partial \bar{K}=\partial\left(\theta_{J}\right. & \wedge \bar{K})+\partial_{J}(\theta \wedge \bar{K}) \\
& =\partial\left(\theta_{J}\right) \wedge \bar{K}+\partial_{J}(\theta) \wedge \bar{K}-\theta_{J} \wedge \partial \bar{K}-\theta \wedge \partial_{J} \bar{K} .
\end{aligned}
$$

The last two terms of (10.2) are equal to $-\theta_{J} \wedge \theta \wedge \bar{K}$ and $-\theta \wedge \theta_{J} \wedge \bar{K}$, therefore they cancel each other. We reduced (10.2) to the equation

$$
0=\partial\left(\theta_{J}\right) \wedge \bar{K}+\partial_{J}(\theta) \wedge \bar{K}
$$

This implies

$$
\partial \theta_{J}+\partial_{J} \theta=0
$$

Using (10.1), we immediately obtain the anticommutation relation

$$
\left\{{ }^{n} \partial,{ }^{n} \partial_{J}\right\}=0 \text {. }
$$

We have checked all the conditions of Theorem 4.3. From Theorem 4.3, we obtain that the $\mathfrak{s l}(2)$-triple $\left\langle L_{\Omega}, \Lambda_{\Omega}, H_{\Omega}\right\rangle$ and the 4-dimensional space

$$
\begin{aligned}
{ }^{n} V:=\left\langle{ }^{n} \partial:=\frac{\partial+\delta^{*}}{2},\right. & { }^{n} \partial_{J}:=\frac{\partial_{J}+\delta_{J}^{*}}{2}, \\
{ }^{n} \partial^{*}: & \left.=\frac{\partial^{*}+\delta^{*}}{2},{ }^{n} \partial_{J}^{*}:=\frac{\partial_{J}^{*}+\delta_{J}}{2}\right\rangle \subset \operatorname{End}\left(\Lambda_{I}^{*, 0}(M)\right) .
\end{aligned}
$$

generate a Kähler-de Rham Lie superalgebra, denoted by ${ }^{n} \mathfrak{g}$.

DEFINITION 10.1. This Lie superalgebra called the normalized Kähler-de Rham superalgebra of an HKT-manifold. 
10.2. The normalized Kähler-de Rham superalgebra and Lefschetz theorem. The normalized Kähler-de Rham superalgebra ${ }^{n} \mathfrak{g}$ has the following geometrical interpretation.

Let $M$ be an HKT-manifold, $\operatorname{dim}_{\mathbb{H}} M=n, I, J, K$ the standard triple of quaternions, $\mathrm{K}=\Lambda_{I}^{2 n, 0}(M)$ the canonical bundle of $(M, I)$ and $\mathfrak{V}=\Omega^{n}$ its nowhere degenerate section provided by the canonical nowhere degenerate $(2,0)$-form $\Omega \in \Lambda_{I}^{2,0}(M)$. Since an HKT-manifold is Hermitian, the bundle $\mathrm{K}$ is equipped with a Hermitian metrics. Consider the standard Hermitian connection $\nabla$ on $\mathrm{K}$ associated with this metrics. Since $\mathfrak{V}$ has constant length, we have

$$
\nabla(\mathfrak{V})=\bar{\partial}(\mathfrak{V})+\overline{\bar{\partial} \mathfrak{V}}
$$

where $\bar{\partial}: \mathrm{K} \longrightarrow \mathrm{K} \otimes \Lambda_{I}^{0,1}(M)$ is the holomorphic structure on $\mathrm{K}$. Interpreting $\mathfrak{V}$ as a $(2 n, 0)$-form on $M$, we find

$$
\bar{\partial} \mathfrak{V}=\bar{\theta} \otimes \mathfrak{V}
$$

where $\theta$ is the canonical $(0,1)$-form defined in Subsection 10.1 (see also Theorem 8.8). Since $\mathfrak{V}$ is a nowhere degenerate section of $K, \mathfrak{V}$ provides a $C^{\infty}$-trivialization of this bundle. Let $\nabla_{0}$ be a flat connection associated with this trivialization. Comparing (10.3) and (10.4), we find that $\nabla$ can be expressed via $\nabla_{0}$ as follows:

$$
\nabla=\nabla_{0}+\theta+\bar{\theta}
$$

In other words, $\theta$ is the $(1,0)$-connection form of the canonical bundle $\mathrm{K}$ associated with the $C^{\infty}$-trivialization provided by the $C^{\infty}$-section $\mathfrak{V} \in \mathrm{K}$.

Let $\mathrm{K}^{1 / 2}$ be a square root of $\mathrm{K}$ determined by the above trivialization. One can define $\mathrm{K}^{1 / 2}$ as a trivial $C^{\infty}$-bundle with a holomorphic structure defined by a connection

$$
\nabla_{1 / 2}=\nabla_{0}+\frac{1}{2} \theta+\frac{1}{2} \bar{\theta}
$$

Consider the (anti-)Dolbeault complex of $\mathrm{K}^{1 / 2}$ :

$$
\mathrm{K}^{1 / 2} \stackrel{\nabla_{1 / 2}^{1,0}}{\longrightarrow} \mathrm{K}^{1 / 2} \otimes \Lambda_{I}^{1,0}(M) \stackrel{\nabla_{1 / 2}^{1,0}}{\longrightarrow} \mathrm{K}^{1 / 2} \otimes \Lambda_{I}^{2,0}(M) \ldots
$$

where $\nabla_{1 / 2}^{1,0}$ is the $(1,0)$-component of $\nabla$. Using the standard trivialization of $\mathrm{K}^{1 / 2}$, we may identify $\mathrm{K}^{1 / 2} \otimes \Lambda_{I}^{*, 0}(M)$ and $\Lambda_{I}^{*, 0}(M)$. By definition of $\nabla_{1 / 2}$, we have

$$
\nabla_{1 / 2}^{1,0}=\partial+\frac{1}{2} \theta
$$

In other words, the twisted Dolbeault differential (10.5) is equal to the normalized HKT differential ${ }^{n} \partial$ of Definition 10.1.

Consider the action of $\mathfrak{s l}(2)=\left\langle L_{\Omega}, \Lambda_{\Omega}, H_{\Omega}\right\rangle$ on the complex (10.5). Since $\mathfrak{s l}(2)$, ${ }^{n} \partial$ and ${ }^{n} \partial^{*}$ are elements of Kähler-de Rham superalgebra, they satisfy the conditions of Theorem 4.3. In particular, the Laplace operator

$$
{ }^{n} \Delta:={ }^{n} \partial^{n} \partial^{*}+{ }^{n} \partial^{* n} \partial
$$


commutes with the $\mathfrak{s l}(2)$-action. On the other hand, the cohomology of (10.5) are identified with the kernel of the Laplacian ${ }^{n} \Delta$. This proves the Lefschetz theorem for the cohomology of the complex (10.5):

ThEOREM 10.2. Let $M$ be a compact HKT-manifold, and $I, J, K$ the standard triple of quaternions, and $\mathrm{K}^{1 / 2}$ be the square root of the canonical class of $(M, I)$ constructed as above. Consider its cohomology space $H^{*}\left(M, \mathrm{~K}^{1 / 2}\right){ }^{1}$ Then

(i) Using the standard trivialization of $\mathrm{K}^{1 / 2}$, we can identify the Dolbeault complex of the bundle $\mathrm{K}^{1 / 2}$ with the complex

$$
0 \longrightarrow \Lambda_{I}^{0,0}(M) \stackrel{{ }^{n} \partial}{\longrightarrow} \Lambda_{I}^{1,0}(M) \stackrel{{ }^{n} \partial}{\longrightarrow} \Lambda_{I}^{2,0}(M) \stackrel{{ }^{n} \partial}{\longrightarrow} \ldots
$$

(ii) The cohomology of $\mathrm{K}^{1 / 2}$, or, what is the same, the cohomology of the complex (10.6), are identified with the kernel of the corresponding Laplacian

$$
{ }^{n} \Delta=\left\{{ }^{n} \partial, \partial^{*}\right\}
$$

(iii) The $\mathfrak{s l}(2)$-triple $\left\langle L_{\Omega}, \Lambda_{\Omega}, H_{\Omega}\right\rangle$ commutes with the action of the normalized Laplacian ${ }^{n} \Delta$.

(iv) This $\mathfrak{s l}(2)$-action provides the "Hard Lefschetz" isomorphism

$$
L_{\Omega}^{n-i}: H^{i}\left(\mathrm{~K}^{1 / 2}\right) \longrightarrow H^{2 n-i}\left(\mathrm{~K}^{1 / 2}\right)
$$

Together with the Serre's duality

$$
H^{2 n-i}\left(\mathrm{~K}^{1 / 2}\right) \cong H^{i}\left(\mathrm{~K}^{1 / 2}\right)^{*}
$$

this gives a canonical isomorphism

$$
H^{i}\left(\mathrm{~K}^{1 / 2}\right) \cong H^{i}\left(\mathrm{~K}^{1 / 2}\right)^{*}
$$

(v) Consider the map

$$
J_{c}: \Lambda_{I}^{p, 0}(M) \longrightarrow \Lambda_{I}^{p, 0}(M)
$$

$J_{c}(\eta)=J(\bar{\eta})$, where $J: \Lambda_{I}^{p, 0}(M) \longrightarrow \Lambda_{I}^{0, p}(M)$ is the standard multiplicative map of differential forms associated with the induced complex structure $J$. Then $J_{c}$ commutes with the normalized Laplacian ${ }^{n} \Delta$.

(vi) The pairing (10.7) can be obtained explicitly as follows. Take $\eta, \eta^{\prime} \in H^{i}\left(\mathrm{~K}^{1 / 2}\right)$ $(i \leqslant n)$. Consider the cohomology class

$$
\eta \wedge J_{c}\left(\eta^{\prime}\right) \in H^{2 i}(M, \mathrm{~K})
$$

and let

$$
\alpha:=L_{\Omega}^{n-i}\left(\eta \wedge J_{c}\left(\eta^{\prime}\right)\right)
$$

be the corresponding element in $H^{2 n}(M, \mathrm{~K}) \cong \mathbb{C}$. Then $\left\langle\eta, \eta^{\prime}\right\rangle=\alpha$, where $\langle\cdot, \cdot\rangle$ is the pairing $(10.7)$.

\footnotetext{
${ }^{1}$ This is the cohomology of the complex (10.5).
} 
10.3. Harmonic spinors on HKT manifolds. We work in assumptions and notations of Theorem 10.2. The canonical class of a hypercomplex Hermitian manifold is topologically trivial: take, for instance, a trivialization associated with the section $\mathfrak{V}$ of the canonical class (Subsection 10.2). Therefore, a hypercomplex Hermitian manifold admits a natural spinor structure.

Let $\mathcal{S}$ be the spinor bundle of $M$. Then $\mathcal{S}$ is isomorphic to $\Lambda_{I}^{*, 0}(M) \otimes \mathrm{K}^{1 / 2}$, where $\mathrm{K}^{1 / 2}$ is the square root of the canonical class constructed above.

It is well known that the Dirac operator corresponds to ${ }^{n} \partial+{ }^{n} \partial^{*}$ under the identification

$$
\Lambda_{I}^{*, 0}(M) \otimes \mathrm{K}^{1 / 2} \cong \mathcal{S}
$$

where ${ }^{n} \partial$ denotes the Dolbeault differential (10.6). Therefore, $H^{*}\left(\mathrm{~K}^{1 / 2}\right)$ is naturally identified with a space of harmonic spinors. Since the harmonic spinors depend from a metric only and not from the choice of a complex structure, we obtain the following corollary.

Corollary 10.3. In assumptions of Theorem 10.2, consider the space $H^{*}\left(\mathrm{~K}^{1 / 2}\right)$. Then $H^{*}\left(\mathrm{~K}^{1 / 2}\right)$ is canonically isomorphic to the space of harmonic spinors. Moreover, $H^{*}\left(\mathrm{~K}^{1 / 2}\right)$ does not depend from the choice of the basis $I, J, K$ in quaternions.

REMARK 10.4. Let $G$ be a compact semisimple Lie group from the list (3.1), equipped with an HKT-structure as in Example 3.6. Then $H^{*}\left(\mathrm{~K}^{1 / 2}\right)=0$. The algebraic-geometrical computation needed for this result was performed by $\mathrm{D}$. Kaledin.

Acknowledgements: This paper appeared as a result of fruitful talks with D. Kaledin. I am grateful to Y.-S. Poon, who told me of HKT-manifolds; A. Losev, who suggested the close study of Lie superalgebras arising from the natural DGalgebras on manifolds; and M. Kontsevich, who told me of M. Reid's conjecture. Many thanks to S. Merkulov for his interest and encouragement and R. Bielawski for interesting discussions. My gratitude to F. Bogomolov and D. Kaledin, who explained me the Bott-Samelson construction. G. Papadopoulos kindly sent some remarks on the history of the subject.

\section{REFERENCES}

[Bes] Besse, A., Einstein Manifolds, Springer-Verlag, New York (1987).

[Bo] Boyer, Charles P., A note on hyper-Hermitian four-manifolds. Proc. Amer. Math. Soc., 102 (1988), no. 1, pp. 157-164.

[Ca] Calabi, E., Metriques kähleriennes et fibrès holomorphes, Ann. Ecol. Norm. Sup., 12 (1979), pp. 269-294.

[CS] CAPria, M. M., SAlamon, S. M., Yang-Mills fields on quaternionic spaces, Nonlinearity, 1 (1988), no. 4 , pp. $517-530$.

[FKS] Figueroa-O'Farrill, J. M., Koehl, C.. Spence, B., Supersymmetry and the cohomology of (hyper)Kähler manifolds, Nuclear Phys. B, 503 (1997), no. 3, pp. 614-626.

[GP] Grantcharov, G., Poon, Y. S., Geometry of hyper-Kähler connections with torsion, Comm. Math. Phys., 213 (2000), no. 1, pp. 19-37.

[GPP] Grantcharov, G., Pedersen H., Poon, Y. S., Lie Groups, Kodaira Manifolds and Complex Deformations, IMADA preprint PP-2001-02, February 7, 2001, 26 pages.

[GH] Griffiths, Ph., HARris, J., Principles of Algebraic Geometry, Wiley-Intersience, New York, 1978. 
[GP1] Gutowski, J., Papadopoulos, G., The Dynamics of Very Special Black Holes, Phys. Lett., B472 (2000), pp. 45-53.

[GP2] Gutowski, J., Papadopoulos, G., The Moduli Spaces of Worldvolume Brane Solitons, Phys. Lett., B432 (1998), pp. 97-102.

[GPS] G.W. Gibbons, G. Papadopoulos, K.S. Stelle, HKT and OKT Geometries on Soliton Black Hole Moduli Spaces, Nucl.Phys., B508 (1997), pp. 623-658.

[HP] Howe, P. S. PaPadopoulos, G., Twistor spaces for hyper-Kähler manifolds with torsion, Phys. Lett., B 379 (1996), no. 1-4, pp. 80-86.

[J] Joyce, Dominic, Compact hypercomplex and quaternionic manifolds, J. Differential Geom., 35 (1992), no. 3, pp. 743-761.

[K] KALEDIN, D., Integrability of the twistor space for a hypercomplex manifold, alggeom/9612016, 9 pages (published in Math. Res. Lett., 1997).

[KV] Kaledin, D., Verbitsky M., Hyperkähler manifolds, Int'l Press, Boston, 2001.

[O] Овата, M., Affine connections on manifolds with almost complex, quaternionic or Hermitian structure, Jap. J. Math., 26 (1955), pp. 43-79.

[OP] Opfermann, A., PaPAdopoulos, G., Homogeneous HKT and QKT manifolds, math$\mathrm{ph} / 9807026$.

[P] Papadopoulos, G., Brane Solitons and Hypercomplex Structures, math.DG/0003024 (published in the volume "Proceedings of the second meeting on "Quaternionic Structures in Mathematics and Physics", World Scientific, 2001).

[PP] Pedersen, H., Poon, Y. S., Inhomogeneous hypercomplex structures on homogeneous manifolds, J. Reine Angew. Math., 516 (1999), pp. 159-181.

[Sa] Samelson, H., A class of complex-analytic manifolds, Portugal. Math., 12 (1953), pp. $129-132$.

[SSTV] Ph. Spindel, A. Sevrin, W. Troost, A. Van Proeyen, Extended supersymmetric $\sigma$ models on group manifolds, Nucl. Phys., B308 (1988), pp. 662-698.

[VO] VERBITSKY M., On the action of a Lie algebra SO(5) on the cohomology of a hyperkähler manifold, Func. Analysis and Appl., 24(2) (1990), pp. 70-71.

[V1] Verbitsky M., Hyperholomorphic bundles over a hyperkähler manifold, alg-geom electronic preprint 9307008 (1993), 43 pages, LaTeX, also published in: Journ. of Alg. Geom., no. 4 (1996), pp. 633-669.

[V2] Veritssky M., Hypercomplex Varieties, alg-geom/9703016 (1997); published in: Comm. Anal. Geom., 7 (1999), no. 2, pp. 355-396.

[V3] Verbitsky M., Projective bundles over hyperkaehler manifolds and stability of FourierMukai transform, math.AG/0107196 (2001), 60 pages. 